\title{
Dressing transformations of constrained Willmore surfaces
}

\author{
Francis E. Burstall and Áurea C. Quintino
}

\begin{abstract}
We use the dressing method to construct transformations of constrained Willmore surfaces in arbitrary codimension. An adaptation of the Terng-Uhlenbeck theory of dressing by simple factors to this context leads us to define Bäcklund transforms of these surfaces for which we prove Bianchi permutability. Specializing to codimension 2, we generalize the Darboux transforms of Willmore surfaces via Riccati equations, due to Burstall-FerusLeschke-Pedit-Pinkall, to the constrained Willmore case and show that they amount to our Bäcklund transforms with real spectral parameter.
\end{abstract}

\section{Introduction}

The Willmore functional is a conformally invariant functional, defined on immersed surfaces in the conformal $n$-sphere, with a long, interesting history $[1,7,21,39,41]$. Its extrema are the Willmore surfaces and these have attracted a lot of attention in recent years, in large part due to interest in the celebrated Willmore conjecture, now affirmed by Marques and Neves [28].

One approach to Willmore surfaces is via a certain Gauss map: to any surface in $S^{n}$, one may associate $[1,7]$ its central sphere congruence. Geometrically, this is a family of 2-spheres tangent to the surface and sharing mean curvature vectors with it. Alternatively, it may be viewed as a map into the space of 2-spheres which is a certain Grassmannian of 4-planes [36]. A key result is that a surface is Willmore if and only if its central sphere congruence is a harmonic map $[1,7,18,35]$ and then the well-developed theory of harmonic maps applies. In particular, the machinery of integrable systems theory becomes available.

The starting point of the integrable systems approach to harmonic maps is the observation $[30,40,42,43]$ that the harmonic map equations amount to the flatness of a family of flat connections depending on a spectral parameter. This structure gives rise to symmetries, of which we shall say much more 
below, and constructions via completely integrable Hamiltonian systems and algebraic curves [11, 23].

This zero curvature representation of the harmonic map equations allows one to deduce two kinds of symmetry. Firstly, harmonic maps admit a spectral deformation [40] by exploiting a scaling freedom in the spectral parameter. Secondly, harmonic maps admit dressing transformations $[38,40]$ which arise by applying carefully chosen gauge transformations to the family of flat connections.

A larger class of surfaces arises when one imposes the weaker requirement that a surface extremize the Willmore functional only with respect to variations which preserve the conformal structure: these are the constrained Willmore surfaces. Now the Euler-Lagrange equations include a Lagrange multiplier in the form of a holomorphic quadratic differential [4] and, while the central sphere congruence is no longer harmonic, a loop of flat connections is still available $[2,14]$.

It is the purpose of the present paper to apply the theory of dressing transformations to constrained Willmore surfaces in $S^{n}$. Two considerations prevent this from being a routine exercise in the existing theory for harmonic maps: first, that theory applies to the central sphere congruence qua map into a Grassmannian and it requires some care to see that the transformed map is the central sphere congruence of a surface at all, let alone a constrained Willmore one. Moreover, the theory must be extended somewhat to cover the possibly non-harmonic central sphere congruences of constrained Willmore surfaces.

To address the second problem, we introduce and study the notion of a $k$-perturbed harmonic map which includes (with $k=2$ ) the central sphere congruences of constrained Willmore surfaces. The idea is that the flat connections associated to harmonic maps are characterized by having simple poles (with respect to the spectral parameter) at 0 and $\infty$. Relaxing this requirement to allow poles of order $k$ leads us to $k$-perturbed harmonic maps. These maps are a geometric incarnation of the $k$ th elliptic integrable system associated with a symmetric space in the sense of Terng [37] (see also Khemar [24]) and also fit into the framework of Brander and Dorfmeister [5]. As such, $k$-perturbed harmonic maps make sense with any symmetric space as target but we focus on the space of 2-spheres that is of immediate interest to us. We define spectral deformations and dressing transformations of such maps, the latter mediated by gauge transformations we call dressing gauges.

We define an energy density for $k$-perturbed harmonic maps which coincides with the harmonic map energy density when $k=1$ and prove that this changes by an explicit exact term under our dressing transformations. 
This result, of possible independent interest, appears to be new, even for harmonic maps.

We then show that our dressing transformations preserve the class of central sphere congruences of constrained Willmore surfaces. In this setting, we see that the Willmore density changes by an exact term only and, moreover, the holomorphic quadratic differential that acts as Lagrange multiplier is fixed by the dressing transformations.

We complete our general theory by adapting the Terng-Uhlenbeck [38] theory of dressing by simple factors to our context. Thus we construct dressing gauges algebraically from parallel subbundles of one of the given flat connections. We call the resulting dressing transformations Bäcklund transformations and prove a Bianchi permutability theorem for them.

Finally, we restrict attention to the case of surfaces in $S^{4}$. Here, there is a well-developed quaternionic formalism $[2,3,10,19]$ that can be brought to bear. In particular, arguing by analogy with Darboux transforms of constant mean curvature surfaces, a Darboux transform of Willmore surfaces in $S^{4}$ is presented in [10] which proceeds by solving a Riccati equation. For us, the key point about this codimension 2 setting is that the space of (oriented) 2 -spheres is a (pseudo-)Hermitian symmetric space. Consequently, one may apply a canonical gauge transformation, arising from the ambient complex structure, to replace the family of flat connections with a simpler untwisted family with poles of about half the order of the original family. Working with the untwisted family, we find that the Bäcklund transformations specialize precisely to simple factor dressing in the sense of Terng-Uhlenbeck and, moreover, can be derived from solutions of a Riccati equation. In particular, we simultaneously extend the Darboux transforms of [10] to the constrained Willmore case and identify them with the Bäcklund transforms with real spectral parameter. These results generalize a similar analysis of harmonic maps into the 2-sphere and the constant mean curvature surfaces of which they are Gauss maps that is carried out in [9].

The results of the paper are based, in part, on those in the second author's PhD thesis $[31,32]$ and some of them were announced in [33].

\section{Constrained Willmore surfaces and perturbed harmonicity}

\subsection{Conformal submanifold geometry}

2.1.1. Conformal geometry of the sphere We are going to study submanifolds of the conformal $n$-sphere $S^{n}$ and find a convenient conformally 
invariant viewpoint on the latter in Darboux's light-cone model [16, Chapitre VI]. For this, contemplate the Lorentzian space-time $\mathbb{R}^{n+1,1}$ with inner product $($,$) and light-cone \mathcal{L}$. The corresponding quadric $\mathbb{P}(\mathcal{L})$ is a conformal manifold: each section $\sigma$ of $\mathcal{L}^{\times} \rightarrow \mathbb{P}(\mathcal{L})$ provides a metric $g_{\sigma}$ via $g_{\sigma}(X, Y)=\left(d_{X} \sigma, d_{Y} \sigma\right)$ and $g_{\mathrm{e}^{u} \sigma}=\mathrm{e}^{2 u} g_{\sigma}$. Fixing unit-timelike $t_{0} \in \mathbb{R}^{n+1,1}$ yields a section $\sigma$ with $\left(\sigma, t_{0}\right) \equiv-1$ and so an isometry $\sigma:\left(\mathbb{P}(\mathcal{L}), g_{\sigma}\right) \cong\{v \in$ $\left.\mathcal{L}:\left(v, t_{0}\right)=-1\right\}$ which last is isometric (via $v \mapsto v-t_{0}$ ) to the unit sphere in $\left\langle t_{0}\right\rangle^{\perp}$. Thus $\mathbb{P}(\mathcal{L}) \cong S^{n}$ qua conformal manifolds.

Moreover, it is clear that the orthogonal group $\mathrm{O}(n+1,1)$ acts transitively and conformally on $\mathbb{P}(\mathcal{L})$. In this way, it actually double covers the Möbius group of global conformal diffeomorphisms on $S^{n}$.

For more details, see [22, Chapter 1].

In the sequel, we will have much to do with $\mathrm{O}(n+1,1)$-connections and so forms with values in the Lie algebra $\mathfrak{o}(n+1,1)$. We shall make repeated (and silent!) use of the isomorphism $\wedge^{2} \mathbb{R}^{n+1,1} \cong o\left(\mathbb{R}^{n+1,1}\right)$ given by $u \wedge v(w):=(u, w) v-(v, w) u$, for $u, v, w \in \mathbb{R}^{n+1,1}$.

\subsubsection{Conformally immersed surfaces and the central sphere con-} gruence A map $f: \Sigma \rightarrow \mathbb{P}(W)$ of a manifold to a projective space is the same as a line subbundle of the trivial bundle $\underline{W}=\Sigma \times W$ : we identify $f$ with the bundle whose fibre at $x$ is $f(x)$. In particular, we will usually view a map $\Lambda: \Sigma \rightarrow \mathbb{P}(\mathcal{L})$ as a null line subbundle of $\underline{\mathbb{R}}^{n+1,1}$. From this point of view, sections of $\Lambda$ are simply lifts of $\Lambda$ to maps $\bar{\Sigma} \rightarrow \mathbb{R}^{n+1,1}$.

Given such a $\Lambda$, we define the derived bundle

$$
\Lambda^{(1)}:=\langle\sigma, d \sigma(T \Sigma)\rangle \leq \Lambda^{\perp}
$$

for $\sigma$ an arbitrary lift of $\Lambda$. Note that $\Lambda$ is an immersion if and only if the bundle $\Lambda^{(1)}$ has rank $\operatorname{dim} \Sigma+1$ and then every lift is also an immersion $\sigma: \Sigma \rightarrow \mathbb{R}^{n+1,1}$.

Henceforth, we will take $\Sigma$ to be a Riemann surface, thus an oriented, conformal manifold of real dimension 2 or, equivalently, a complex manifold of dimension 1. We denote the complex structure of $\Sigma$ by $J^{\Sigma}$. The type decomposition of $T \Sigma$ now induces a decomposition of $\Lambda^{(1)} / \Lambda$ : set

$$
\Lambda^{1,0}=\Lambda \oplus d \sigma\left(T^{1,0} \Sigma\right) \quad \Lambda^{0,1}=\Lambda \oplus d \sigma\left(T^{0,1} \Sigma\right)
$$

for some, hence any, lift $\sigma$. Then

$$
\Lambda^{1,0}+\Lambda^{0,1}=\Lambda^{(1)}
$$




$$
\begin{aligned}
\Lambda^{1,0} \cap \Lambda^{0,1} & =\Lambda, \\
\Lambda^{1,0} & =\overline{\Lambda^{0,1}} .
\end{aligned}
$$

Convention. Here and below, we do not distinguish notationally between a subbundle and its complexification. In fact, we shall usually take all subbundles to be complex subbundles of $\underline{\mathbb{C}}^{n+2}=\left(\underline{\mathbb{R}}^{n+1,1}\right)^{\mathbb{C}}$ and view such a subbundle as real if it is stable under the complex conjugation on $\underline{\mathbb{C}}^{n+2}$ with fixed set $\underline{\mathbb{R}}^{n+1,1}$.

An immersion $\Lambda$ is conformal if and only if each lift is a conformal immersion $\Sigma \rightarrow \mathbb{R}^{n+1,1}$ if and only if $\Lambda^{1,0}$ (and hence also $\Lambda^{0,1}$ ) is isotropic with respect to the (complex bilinear extension of) $($,$) .$

A key tool in conformal submanifold geometry is the central sphere congruence or conformal Gauss map $[1,7,18,21,35] V$ of a conformal immersion $\Lambda$. For us, this is the bundle $V \leq \underline{\mathbb{R}}^{n+1,1}$ of $(3,1)$-planes given by

$$
\Lambda^{(1)} \oplus\langle\Delta \sigma\rangle
$$

for $\sigma$ any lift of $\Lambda$ and $\Delta$ the Laplacian of $g_{\sigma}$.

From $V$, we get an orthogonal decomposition of the trivial bundle

$$
\underline{\mathbb{R}}^{n+1,1}=V \oplus V^{\perp}
$$

and a corresponding reduction of the trivial connection:

$$
d=\mathcal{D}_{V}+\mathcal{N}_{V}
$$

where $V, V^{\perp}$ are $\mathcal{D}_{V}$-parallel and $\mathcal{N}_{V}$ takes values in $V \wedge V^{\perp} \subset \mathfrak{o}\left(\underline{\mathbb{R}}^{n+1,1}\right)$. Alternatively, viewing $V$ as a map $\Sigma \rightarrow \operatorname{Gr}_{(3,1)}\left(\mathbb{R}^{n+1,1}\right)$ to the Grassmannian of $(3,1)$-planes, $\mathcal{N}_{V}$ may be canonically identified with $d V$.

We note that since $\Lambda^{(1)} \leq V, \mathcal{N}_{V}(\Lambda)=0$ and, similarly, $\Delta \sigma \in \Gamma V$ is equivalent to $\mathcal{N}_{V}^{1,0}\left(\Lambda^{0,1}\right)=0$.

\subsection{Constrained Willmore surfaces and holomorphic quadric differentials}

Let $\Lambda$ be a conformal immersion of a compact Riemann surface $\Sigma$ with central sphere congruence $V$. The Willmore energy $W(\Lambda)$ of $\Lambda$ is given by

$$
W(\Lambda)=\frac{1}{2} \int_{\Sigma}\left|\Pi_{0}\right|^{2}
$$


where $\Pi_{0}$ is the trace-free second fundamental form of $\Lambda$ (computed against any representative metric on $S^{n}$ and independent of that choice). This is known $[1,7,10,13,18]$ to coincide with the harmonic map energy of $V$ :

$$
W(\Lambda)=E(V)=\frac{1}{2} \int_{\Sigma}\left(\mathcal{N}_{V} \circ J^{\Sigma} \wedge \mathcal{N}_{V}\right)
$$

A conformal immersion is Willmore if it extremizes the Willmore functional and constrained Willmore if it extremizes the Willmore functional with respect to infinitesimally conformal variations. It follows at once from (2.1) that a surface is Willmore when its central sphere congruence is harmonic and it is well known $[1,7,35]$ that the converse holds. Thus, a surface is Willmore if and only if $d^{\mathcal{D}} * \mathcal{N}=0$. More generally, we have the following reformulation of a theorem of Bohle-Peters-Pinkall [4]:

Theorem 2.1 [13, Section 14]. $\Lambda$ is a constrained Willmore surface if and only if there exists a real form $q \in \Omega^{1}\left(\Lambda \wedge \Lambda^{(1)}\right)$ with

$$
d^{\mathcal{D}_{V}} q=0
$$

such that

$$
d^{\mathcal{D}_{V}} * \mathcal{N}_{V}=2\left[q \wedge * \mathcal{N}_{V}\right]
$$

Such a form $q$ is said to be a (Lagrange) multiplier for $\Lambda$. Thus Willmore surfaces are constrained Willmore surfaces with zero multiplier.

When $\Sigma$ is non-compact, we define constrained Willmore surfaces to be the solutions of $(2.2)$.

The 1-form $q$ is a bundle-valued avatar of a more familiar geometric object: a holomorphic quadratic differential on $\Sigma$. Indeed, given $q \in \Omega^{1}(\Lambda \wedge$ $\left.\Lambda^{(1)}\right)$, we define a 2-tensor $Q$ on $M$ by setting

$$
Q(X, Y) \sigma:=q_{X}\left(d_{Y} \sigma\right)
$$

for $\sigma \in \Gamma \Lambda$. We then have

Lemma 2.2 [13, Section 12]. Let $q \in \Omega^{1}\left(\Lambda \wedge \Lambda^{(1)}\right)$ and define $Q$ by (2.3). Then $d^{\mathcal{D}_{V}} q=0$ if and only if $Q$ is symmetric and trace-free, thus $Q^{1,1}=0$, and $Q^{2,0}$ is holomorphic.

In this case, $q^{1,0} \in \Omega^{1}\left(\Lambda \wedge \Lambda^{0,1}\right)$. 
It is the holomorphic quadratic differential $Q$ that appears in the BohlePeters-Pinkall version of $(2.2 \mathrm{~b})$.

Remark 2.3. In general, for a given constrained Willmore surface $\Lambda$, the multiplier $q$ is unique. The exception is when $\Lambda$ is in addition isothermic: if $d^{\mathcal{D}_{V}} * \mathcal{N}_{V}=2\left[q_{1} \wedge * \mathcal{N}_{V}\right]=2\left[q_{2} \wedge * \mathcal{N}_{V}\right]$ with $d^{\mathcal{D}_{V}} q_{i}=0$ then $\eta:=* q_{2}-* q_{1}$ solves $d^{\mathcal{D}_{V}} \eta=\left[\mathcal{N}_{V} \wedge \eta\right]=0$ or, equivalently, $d \eta=0$. This last condition amounts to the existence of a holomorphic quadratic differential that commutes with the trace-free second fundamental form, that is, that $\Lambda$ is isothermic. In this situation, $q_{1}+* s \eta$ is a multiplier for $\Lambda$ for each $s \in \mathbb{R}$.

The isothermic Willmore surfaces in $S^{3}$ are precisely the minimal surfaces with respect to a constant curvature metric [39] while the isothermic constrained Willmore surfaces include those of constant mean curvature [34]. See [4, 14] for more details on this topic.

\subsection{Flat connections and perturbed harmonic maps}

The key to the integrable systems approach to harmonic maps is the wellknown observation that the harmonic map equations amount to the flatness of a family of connections [30, 40, 42, 43]. In particular, this gives a zero-curvature characterization of Willmore surfaces but more is true: the constrained Willmore equations also admit a spectral deformation [14] and hence a zero-curvature representation:

Theorem 2.4 [13]. A conformal immersion $\Lambda$ with central sphere congruence $V$ is constrained Willmore if and only if there exists a real form $q \in \Omega^{1}\left(\Lambda \wedge \Lambda^{(1)}\right)$ such that

$$
d^{\lambda}:=\mathcal{D}_{V}+\lambda \mathcal{N}_{V}^{1,0}+\lambda^{-1} \mathcal{N}_{V}^{0,1}+\left(\lambda^{2}-1\right) q^{1,0}+\left(\lambda^{-2}-1\right) q^{0,1}
$$

is flat for all $\lambda \in \mathbb{C}^{\times}$.

Let us abstract the main properties enjoyed by $V$ and the family of connections $d^{\lambda}$. For this, we take $V \leq \underline{\mathbb{R}}^{n+1,1}$ to be any bundle of $(3,1)$-planes and consider the corresponding decomposition of the trivial connection: $d=$ $\mathcal{D}_{V}+\mathcal{N}_{V}$. Let $\rho_{V}$ denote reflection across $V$.

Definition 2.5. For $k \in \mathbb{N}$, say that $V$ is $k$-perturbed harmonic if there is a family of flat metric connections $d^{\lambda}, \lambda \in \mathbb{C}^{\times}$, on $\underline{\mathbb{C}}^{n+2}$, with the following 
properties:

$$
\begin{aligned}
d^{1} & =d & & \text { (normalization) }, \\
\rho_{V} \cdot d^{\lambda} & =d^{-\lambda} & & \left(\rho_{V} \text {-twisted }\right), \\
d^{1 / \bar{\lambda}} & =\overline{d^{\lambda}} & & \text { (reality) }
\end{aligned}
$$

and $\lambda \mapsto\left(d^{\lambda}\right)^{1,0}$ is holomorphic on $\mathbb{C}$ with a pole of order $k$ at $\infty$ (whence, thanks to $(2.5 \mathrm{c}), \lambda \mapsto\left(d^{\lambda}\right)^{0,1}$ is holomorphic on $\mathbb{C}^{\times} \cup\{\infty\}$ with a pole of order $k$ at 0$)$. In this case, we say that $\left(V, d^{\lambda}\right)$ is a $k$-perturbed harmonic bundle. ${ }^{1}$

In this situation, we may write

$$
d^{\lambda}=\sum_{|i| \leq k} \lambda^{i} A_{i}
$$

with $A_{0}$ a real metric connection preserving $V$ and $A_{i} \in \Omega^{1}\left(\mathfrak{o}\left(\underline{\mathbb{C}}^{n+2}\right)\right), i \neq 0$, satisfying $A_{i}^{1,0}=0$, for $i<0 ; A_{-i}=\overline{A_{i}} ; \operatorname{Ad}_{\rho_{V}} A_{i}=(-1)^{i} A_{i}$ and

$$
\mathcal{D}_{V}=\sum_{i} A_{2 i} \quad \mathcal{N}_{V}=\sum_{i} A_{2 i+1} .
$$

It follows that $V$ is 1-perturbed harmonic if and only if it is harmonic $\left(A_{0}=\right.$ $\mathcal{D}_{V}$ and $\left.A_{1}=\mathcal{N}_{V}^{1,0}\right)$.

Notation. For $\lambda \in \mathbb{C} \cup\{\infty\}$, set $\lambda^{*}=1 / \bar{\lambda}$ so that the reality condition (2.5c) reads

$$
d^{\lambda^{*}}=\overline{d^{\lambda}}
$$

With these notions in hand, we reformulate Theorem 2.4 as follows:

Theorem 2.6. A conformal immersion $\Lambda$ with central sphere congruence $V$ is constrained Willmore if and only if $V$ is 2-perturbed harmonic with $d^{\lambda}$ satisfying $A_{2}+A_{-2} \in \Omega^{1}\left(\Lambda \wedge \Lambda^{(1)}\right)$.

Remark 2.7. The condition of k-perturbed harmonicity makes sense for maps into an arbitrary Grassmannian of non-degenerate subspaces of an inner product space $\mathbb{R}^{p, q}$, or, indeed, an arbitrary pseudo-Riemannian symmetric space. Much of what we say below applies in this more general context.

\footnotetext{
${ }^{1} \mathrm{~A}$ little circumspection is required here as we have seen in Remark 2.3 that a given bundle $V$ can be $k$-perturbed harmonic in more than one way.
} 


\section{Transformations of $k$-perturbed harmonic bundles and constrained Willmore surfaces}

Our $k$-perturbed harmonic bundles come equipped with a family of flat connections $d^{\lambda}$. Such connections, at least on simply connected open subsets of $\Sigma$, are gauge equivalent to the trivial connection. These, and other, gauge transformations can be exploited to produce new $k$-perturbed harmonic bundles in various ways. We begin with the simplest of these transformations.

\subsection{Spectral deformation}

Let $\left(V, d^{\lambda}\right)$ be $k$-perturbed harmonic. For $\mu \in S^{1}$, we have from $(2.5 \mathrm{c})$ that $d^{\mu}$ is a real, flat, metric connection and so there is, at least locally, a gauge transformation $\Phi_{\mu} \in \Gamma\left(\mathrm{O}\left(\underline{\mathbb{R}}^{n+1,1}\right)\right)$ with

$$
\Phi_{\mu} \cdot d^{\mu}=d
$$

where $\Phi_{\mu} \cdot d^{\mu}=\Phi_{\mu} \circ d^{\mu} \circ \Phi_{\mu}^{-1}$ is the usual action of gauge transformations on connections. We note that $\Phi_{\mu}$ is unique up to left multiplication by a constant element of $\mathrm{O}(n+1,1)$.

Proposition 3.1. Define $V_{\mu}:=\Phi_{\mu} V$. Then $V_{\mu}$ is also k-perturbed harmonic with associated flat connections

$$
d_{\mu}^{\lambda}=\Phi_{\mu} \cdot d^{\lambda \mu}
$$

Proof. The connections $d_{\mu}^{\lambda}$ are certainly flat, being gauges of the flat connections $d^{\lambda \mu}$, so we must show that they have the properties enumerated in Definition 2.5. First, (3.1) tells us that $d_{\mu}^{1}=d$ so the family is normalized. Secondly, we have $\rho_{V_{\mu}} \circ \Phi_{\mu}=\Phi_{\mu} \circ \rho_{V}$ which, along with (2.5b), rapidly yields that $d_{\mu}^{\lambda}$ is $\rho_{V_{\mu}}$-twisted. Similarly, $\mu=\mu^{*}$ and $\overline{\Phi_{\mu}}=\Phi_{\mu}$ which, together with $(2.5 \mathrm{c})$, gives the reality condition for $d_{\mu}^{\lambda}$. Finally, $\lambda \mapsto d^{\lambda \mu}$ has the same poles at 0 and $\infty$ as $d^{\lambda}$ whence $\lambda \mapsto d_{\mu}^{\lambda}$ does also.

We can iterate this construction: for $\nu \in S^{1}$, find a gauge transformation $\Phi_{\nu}^{\mu}$ for which $\Phi_{\nu}^{\mu} \cdot d_{\mu}^{\nu}=d$. Together with (3.2), this gives

$$
\left(\Phi_{\nu}^{\mu} \Phi_{\mu}\right) \cdot d^{\mu \nu}=d
$$

so that we can take $\Phi_{\mu \nu}=\Phi_{\nu}^{\mu} \Phi_{\mu}$ and conclude: 
Proposition 3.2. For $\mu, \nu \in S^{1}$ and $\left(V, d^{\lambda}\right)$ k-perturbed harmonic, $V_{\mu \nu}=$ $\left(V_{\mu}\right)_{\nu}$.

Thus we have an $S^{1}$-action on $k$-perturbed harmonic bundles up to congruence. The analysis applies for $k$-perturbed harmonic maps into any symmetric space, mutatis mutandis, and, for $k=1$, recovers the well-known result of Terng (cf. [40]).

Expanding (3.2) in powers of $\lambda$ gives:

Lemma 3.3. In the situation of Proposition 3.1, write $d^{\lambda}=\sum A_{i}$ and $d_{\mu}^{\lambda}=$ $\sum A_{i}^{\mu}$. Then

$$
\begin{aligned}
& A_{0}^{\mu}=\Phi_{\mu} \cdot A_{0}, \\
& A_{i}^{\mu}=\mu^{i} \operatorname{Ad}_{\Phi_{\mu}} A_{i},
\end{aligned}
$$

for $i \neq 0$.

We use this to examine the special case where $V$ is the central sphere congruence of a constrained Willmore surface $\Lambda$ with multiplier $q$. In this case, set $\Lambda_{\mu}=\Phi_{\mu} \Lambda$ and define $q_{\mu}$ by

$$
q_{\mu}=A_{2}^{\mu}+A_{-2}^{\mu}=\operatorname{Ad}_{\Phi_{\mu}}\left(\mu^{2} q^{1,0}+\mu^{-2} q^{0,1}\right)
$$

where the last equality is an instance of $(3.3 \mathrm{~b})$. We now recover a result of [14] as formulated in [13]:

Proposition 3.4 [14]. For each $\mu \in S^{1}, \Lambda_{\mu}$ is a conformal immersion and a constrained Willmore surface with multiplier $q_{\mu}$.

Proof. To see that $\Lambda_{\mu}$ is a conformal immersion, we need $\Lambda_{\mu}^{1,0}$ to be maximal isotropic in $V_{\mu}$. For $V_{\mu}$ to be the central sphere congruence of $\Lambda_{\mu}$, we need $\mathcal{N}_{V_{\mu}}^{1,0} \Lambda_{\mu}^{0,1}=0$ and, finally, we need $q_{\mu}^{1,0}$ to take values in $\wedge^{2} \Lambda_{\mu}^{0,1}$. Then Theorem 2.6 yields the result. However, since $(3.3 \mathrm{~b})$ gives $\mathcal{N}_{V_{\mu}}^{1,0}=\mu \operatorname{Ad}_{\Phi_{\mu}} \mathcal{N}_{V}^{1,0}$, all of this follows as soon as we know that $\Lambda_{\mu}^{1,0}=\Phi_{\mu} \Lambda^{1,0}$. For this last, observe that $q \Lambda=\mathcal{N} \Lambda=0$, so that the operators $d^{\mu}$ and $d$ coincide on $\Gamma \Lambda$. We conclude that $\Lambda^{1,0}=\Lambda \oplus d^{\mu} \sigma\left(T^{1,0} \Sigma\right)$, for any $\sigma \in \Gamma \Lambda$ and, gauging by $\Phi_{\mu}$, we see that $\Lambda_{\mu}^{1,0}=\Phi_{\mu} \Lambda^{1,0}$ as required. 


\subsection{Dressing action}

We are going to use a version of the Terng-Uhlenbeck dressing action [38] to construct new constrained harmonic bundles from $V$. The key idea here is to find $\lambda$-dependent gauge transformations that preserve the algebraic shape of $d^{\lambda}$ (see $[12,15]$ for similar viewpoints on dressing). These transformations, when applied to the central sphere congruence of a constrained Willmore surface, will give rise to new such surfaces.

We begin by specifying the properties of this gauge transformation.

Definition 3.5. Let $\left(V, d^{\lambda}\right)$ be a $k$-perturbed harmonic bundle. A dressing gauge for $\left(V, d^{\lambda}\right)$ is a family of gauge transformations $\lambda \mapsto r(\lambda) \in \Gamma \mathrm{O}\left(\underline{\mathbb{C}}^{n+2}\right)$ which is holomorphic in $\lambda$ near $0, \infty \in \mathbb{P}^{1}$ and has the following properties:

(1) $r(-\lambda) \circ \rho_{V} \circ r(\lambda)^{-1}$ is independent of $\lambda \in \operatorname{dom}(r)$.

(2) For all $\lambda \in \operatorname{dom}(r)$,

$$
r\left(\lambda^{*}\right)=\overline{r(\lambda)} \quad(\text { reality })
$$

(Thus we require that $\operatorname{dom}(r)$ is stable under $\lambda \mapsto \pm \lambda^{*}$.)

(3) The connections $r(\lambda) \cdot d^{\lambda}$ extend from $\operatorname{dom}(r) \backslash\{0, \infty\}$ to a holomorphic family of connections $\hat{d}^{\lambda}$ on $\mathbb{C}^{\times}$with $\hat{d}^{1}=d$.

$$
\operatorname{det}\left(r(0)^{-1} r(\infty)_{\mid V}\right)=1
$$

For such an $r$, set $\hat{V}=r(0) V$ and call $\left(\hat{V}, \hat{d}^{\lambda}\right)$ the dressing transform of $\left(V, d^{\lambda}\right)$ by $r$.

Note that item (1) now reads

$$
r(-\lambda) \circ \rho_{V}=\rho_{\hat{V}} \circ r(\lambda), \quad \text { (twisted) }
$$

for $\lambda \in \operatorname{dom}(r)$, and then evaluating at $\lambda=\infty$ yields $\hat{V}=r(\infty) V$ also. In particular, $r(0)^{-1} r(\infty) V=V$, so that (3.5) makes sense.

It is our contention that $\left(\hat{V}, \hat{d}^{\lambda}\right)$ is again a $k$-perturbed harmonic map $\Sigma \rightarrow \operatorname{Gr}_{3,1}\left(\mathbb{R}^{n+1,1}\right)$. We begin by showing that $\hat{V}$ is indeed (the complexification of ) a bundle of real $(3,1)$-planes. First, evaluate (3.4) at $\lambda=0$, to get $r(\infty)=\overline{r(0)}$ so that $\overline{\hat{V}}=r(\infty) V=\hat{V}$. We now have: 
Lemma 3.6. Let $W \leq \mathbb{C}^{n+2}$ be a real (thus $W=\bar{W}$ ), non-degenerate 4plane and $T \in \mathrm{O}\left(\mathbb{C}^{n+2}\right)$. Then

(1) $W \cap \mathbb{R}^{n+1,1}$ is a $(3,1)$-plane if and only if $\operatorname{dim}(U \cap \bar{U})=1$, for any maximal isotropic 2-plane $U \leq W$.

(2) If $W \cap \mathbb{R}^{n+1,1}$ is a $(3,1)$-plane and $T W=\overline{T W}$ then $T W \cap \mathbb{R}^{n+1,1}$ is also $a(3,1)$-plane if and only if $\operatorname{det}\left(T^{-1} \bar{T}\right)=1$.

Proof. For (1), note that $W_{\mathbb{R}}:=W \cap \mathbb{R}^{n+1,1}$ has signature $(4,0)$ or $(3,1)$. Since $U \cap \bar{U} \cap \mathbb{R}^{n+1,1}$ is a real isotropic subspace of $W_{\mathbb{R}}$, we have $\operatorname{dim}(U \cap$ $\bar{U}) \leq 1$ with equality forcing $W_{\mathbb{R}}$ to have signature $(3,1)$. For the converse, if $\operatorname{dim}(U \cap \bar{U})=0$, then $U$ and $\bar{U}$ are the $\pm \sqrt{-1}$-eigenspaces of an orthogonal complex structure on $W_{\mathbb{R}}$ and this requires that $W_{\mathbb{R}}$ be a $(4,0)$-plane.

For (2), let $U \leq W$ be maximal isotropic so that $T U$ is maximal isotropic in $T W$. We have just seen that $(T W)_{\mathbb{R}}$ is a $(3,1)$-plane if and only if $\operatorname{dim}(T U \cap \overline{T U})=1$, or, equivalently, $\operatorname{dim} U \cap\left(T^{-1} \bar{T}\right) \bar{U}=1$. Now $U$ and $\bar{U}$ define lines in the quadric in $\mathbb{P}(W)$ given by the ambient inner product and such lines intersect exactly when they lie in different rulings of that quadric. These rulings comprise different $\mathrm{SO}(W)$-orbits which are permuted by $\mathrm{O}(W) \backslash \mathrm{SO}(W)$. Thus $\operatorname{det}\left(T^{-1} \bar{T}\right)=1$ if and only if $\bar{U}$ and $\left(T^{-1} \bar{T}\right) \bar{U}$ lie in the same ruling if and only if $\left(T^{-1} \bar{T}\right) \bar{U}$ intersects $U$ in a single (projective) point.

Applying this last fibrewise to $V$ with $T=r(0)$ and using (3.5) immediately yields:

Corollary 3.7. Let $\left(\hat{V}, \hat{d}^{\lambda}\right)$ be the dressing transform of $\left(V, d^{\lambda}\right)$ by $r$. Then,

(1) $\overline{\hat{V}}=\hat{V}$.

(2) $\hat{V} \cap \underline{\mathbb{R}}^{n+1,1}$ is a bundle of (3,1)-planes.

(3) For any maximal isotropic subbundle of $U$ of $\hat{V}, U \cap \bar{U}$ has rank 1.

With these preliminaries dealt with, we have:

Theorem 3.8. Let $\left(\hat{V}, \hat{d}^{\lambda}\right)$ be the dressing transform of $\left(V, d^{\lambda}\right)$ by $r$. Then $\left(\hat{V}, \hat{d}^{\lambda}\right)$ is k-perturbed harmonic.

Proof. This amounts to showing that the connections $\hat{d}^{\lambda}$ satisfy the conditions of Definition 2.5. By hypothesis, $\hat{d}^{\lambda}$ is normalized. For the remaining conditions that are pointwise in $\lambda$, we establish them first for $\lambda \in \operatorname{dom}(r) \backslash$ 
$\{0, \infty\}$ and then conclude they hold for all $\lambda \in \mathbb{C}^{\times}$by unique continuation. Indeed, (3.6) together with the fact that $d^{\lambda}$ is $\rho_{V}$-twisted combine to show that $\hat{d}^{\lambda}$ is $\rho_{\hat{V}}$-twisted on $\operatorname{dom}(r) \backslash\{0, \infty\}$. Similarly, the reality condition on $\hat{d}^{\lambda}$ follows immediately from that on $d^{\lambda}$ and (3.4). Moreover, for $\lambda \in \operatorname{dom}(r) \backslash\{0, \infty\}, \hat{d}^{\lambda}$ is flat since it is a gauge of the flat connection $d^{\lambda}$.

Finally, the pole behaviour of $\hat{d}^{\lambda}$ at zero and infinity coincides with that of $d^{\lambda}$ since the connections differ by the gauge transformations $r(\lambda)$ which are holomorphic in $\lambda$ near those points.

To go further, we need to establish some relations between the coefficients $\hat{A}_{i}$ of $\hat{d}^{\lambda}$ and those of $d^{\lambda}$. First let us establish some notation: write

$$
A_{+}=\sum_{0<i \leq k} \lambda^{i} A_{i}, \quad A_{-}=\sum_{k \leq i<0} \lambda^{i} A_{i}
$$

so that $d^{\lambda}=A_{+}+A_{0}+A_{-}$and, similarly, $\hat{d}^{\lambda}=\hat{A}_{+}+\hat{A}_{0}+\hat{A}_{-}$. Moreover, define $\chi_{0}, \chi_{\infty}$ by

$$
\begin{gathered}
\chi_{0}=r^{-1} \partial r / \partial \lambda, \\
\chi_{\infty}=r^{-1} \partial r / \partial \mu,
\end{gathered}
$$

where $\mu=1 / \lambda$. We note that $\chi_{\infty}(\lambda)=\overline{\chi_{0}\left(\lambda^{*}\right)}$, for $\lambda \in \mathbb{C}^{\times}$. On $\operatorname{dom}(r) \backslash$ $\{0, \infty\}$, we have

$$
\hat{d}^{\lambda}=r(\lambda) \cdot d^{\lambda}
$$

The (1,0)-part of this is holomorphic at $\lambda=0$ and differentiating with respect to $\lambda$ yields

$$
\partial \hat{A}_{+} / \partial \lambda=\operatorname{Ad}_{r}\left(\partial A_{+} / \partial \lambda-A_{0}^{1,0} \chi_{0}-\left[A_{+}, \chi_{0}\right]\right)
$$

Again, we may write the $(0,1)$-part of $(3.7)$ as

$$
\hat{A}_{0}^{0,1}+\hat{A}_{-}=r \cdot A_{0}^{0,1}+\operatorname{Ad}_{r} A_{-}
$$

from which we conclude that $\hat{A}_{-}-\operatorname{Ad}_{r} A_{-}$is holomorphic near $\lambda=0$. 
Taking coefficients of powers of $\lambda$ now yields:

Lemma 3.9. In the situation of Theorem 3.8, with $d^{\lambda}=\sum_{|i| \leq k} \lambda^{i} A_{i}$ and $\hat{d}^{\lambda}=\sum_{|i| \leq k} \lambda^{i} \hat{A}_{i}$, we have:

$$
\hat{A}_{0}^{1,0}=r(0) \cdot A_{0}^{1,0}, \quad \hat{A}_{0}^{0,1}=r(\infty) \cdot A_{0}^{0,1},
$$

$$
\hat{A}_{1}=\operatorname{Ad}_{r(0)}\left(A_{1}-A_{0}^{1,0} \chi_{0}(0)\right), \quad \hat{A}_{-1}=\operatorname{Ad}_{r(\infty)}\left(A_{-1}-A_{0}^{0,1} \chi_{\infty}(\infty)\right),
$$

$$
\hat{A}_{-k}=\operatorname{Ad}_{r(0)} A_{-k}, \quad \hat{A}_{k}=\operatorname{Ad}_{r(\infty)} A_{k}
$$

and, for $k>1$,

$$
\begin{aligned}
\hat{A}_{-k+1} & =\operatorname{Ad}_{r(0)}\left(A_{-k+1}+\left[\chi_{0}(0), A_{-k}\right]\right) \\
\hat{A}_{k-1} & =\operatorname{Ad}_{r(\infty)}\left(A_{k-1}+\left[\chi_{\infty}(\infty), A_{k}\right]\right) .
\end{aligned}
$$

Finally,

$$
\begin{aligned}
& \hat{A}_{0}^{1,0}=r(\infty) \cdot A_{0}^{1,0}+\frac{1}{k !} \frac{\partial^{k}}{\partial \mu^{k}}{ }_{\mid \mu=0} \operatorname{Ad}_{r}\left(A_{k}+\cdots+\mu^{k-1} A_{1}\right), \\
& \hat{A}_{0}^{0,1}=r(0) \cdot A_{0}^{0,1}+\frac{1}{k !} \frac{\partial^{k}}{\partial \lambda^{k}}{ }_{\mid \lambda=0} \operatorname{Ad}_{r}\left(A_{-k}+\cdots+\lambda^{k-1} A_{-1}\right) .
\end{aligned}
$$

Proof. Evaluate the $(1,0)$ part of $(3.7)$ at $\lambda=0$ to get $(3.10 a)^{1,0}$ and evaluate (3.8) at $\lambda=0$ to get $(3.10 \mathrm{~b})^{1,0}$.

Meanwhile, comparing coefficients of $\lambda$ in (3.9) yields

$$
\begin{aligned}
\hat{A}_{-k+i} & =\frac{1}{i !} \frac{\partial^{i}}{\partial \lambda^{i}}{ }_{\mid \lambda=0} \operatorname{Ad}_{r(\lambda)}\left(A_{-k}+\cdots+\lambda^{k-1} A_{-1}\right), \text { for } 0 \leq i<k, \\
\hat{A}_{0}^{0,1} & =r(0) \cdot A_{0}^{0,1}+\frac{1}{k !} \frac{\partial^{k}}{\partial \lambda^{k}}{ }_{\mid \lambda=0} \operatorname{Ad}_{r(\lambda)}\left(A_{-k}+\cdots+\lambda^{k-1} A_{-1}\right) .
\end{aligned}
$$

The last equation is $(3.10 \mathrm{e})^{0,1}$ while the cases $i=0,1$ of the first yield $(3.10 \mathrm{c})^{0,1}$ and $(3.10 \mathrm{~d})^{0,1}$. A similar argument at $\mu=0$ (or an appeal to the reality of $\hat{d}^{\lambda}$ ) gives the remaining equations.

We pause for a short diversion of possibly independent interest: our dressing transformation changes a certain energy density by an exact 2form. First a definition: 
Definition 3.10. Let $\left(V, d^{\lambda}\right)$ be $k$-perturbed harmonic. The energy density $e(V)$ of $\left(V, d^{\lambda}\right)$ is the 2 -form on $\Sigma$ given by

$$
e(V)=i \sum_{0<j \leq k} j\left(A_{j} \wedge A_{-j}\right)
$$

Note that, when $V$ is harmonic (thus $k=1$ ), $e(V)$ is the usual harmonic map energy density:

$$
e(V)=\frac{1}{2}\left(\mathcal{N}_{V} \circ J^{\Sigma} \wedge \mathcal{N}_{V}\right)
$$

We now have:

Proposition 3.11. Let $\left(V, d^{\lambda}\right)$ and $\left(\hat{V}, \hat{d}^{\lambda}\right)$ be $k$-perturbed harmonic bundles with $\hat{V}$ the dressing transform of $V$ by $r$. Then

$$
e(\hat{V})=e(V)-\mathrm{i} d \operatorname{Res}_{\lambda=0}\left(\chi_{0}, A_{-}\right) .
$$

Proof. We begin by observing that our energy density is a residue:

$$
\sum_{0<j \leq k} j\left(A_{j} \wedge A_{-j}\right)=\operatorname{Res}_{\lambda=0}\left(\partial A^{+} / \partial \lambda \wedge A^{-}\right) .
$$

Now $\hat{A}^{-}-\operatorname{Ad}_{r} A^{-}$is holomorphic near $\lambda=0$ so that (3.8) yields:

$$
\begin{aligned}
\operatorname{Res}_{\lambda=0}\left(\partial \hat{A}^{+} / \partial \lambda \wedge \hat{A}^{-}\right) & =\operatorname{Res}_{\lambda=0}\left(\operatorname{Ad}_{r}\left(\partial A^{+} / \partial \lambda-A_{0}^{1,0} \chi_{0}-\left[A_{+}, \chi_{0}\right]\right) \wedge \operatorname{Ad}_{r} A^{-}\right) \\
& =\operatorname{Res}_{\lambda=0}\left(\partial A^{+} / \partial \lambda-A_{0}^{1,0} \chi_{0}-\left[A_{+}, \chi_{0}\right] \wedge A^{-}\right) .
\end{aligned}
$$

Since $A_{-}$is a $(0,1)$-form, $\left(A_{0}^{1,0} \wedge A_{-}\right)=\left(A_{0} \wedge A_{-}\right)$and we have

$$
\begin{aligned}
\left(A_{0}^{1,0} \chi_{0}+\left[A_{+}, \chi_{0}\right] \wedge A^{-}\right) & =\left(A_{0} \chi_{0}+\left[A_{+}, \chi_{0}\right] \wedge A^{-}\right) \\
& =d\left(\chi_{0}, A_{-}\right)-\left(\chi_{0}, d^{A_{0}} A_{-}\right)-\left(\chi_{0},\left[A_{+} \wedge A_{-}\right]\right) \\
& =d\left(\chi_{0}, A_{-}\right)+\left(\chi_{0}, R^{A_{0}}+d^{A_{0}} A_{+}\right)
\end{aligned}
$$

where, for the last equality, we have used the flatness of $d^{\lambda}$. Since $\left(\chi_{0}, R^{A_{0}}+\right.$ $\left.d^{A_{0}} A_{+}\right)$is holomorphic near $\lambda=0$ and so has no residue there, we conclude:

$$
\operatorname{Res}_{\lambda=0}\left(\partial \hat{A}^{+} / \partial \lambda \wedge \hat{A}^{-}\right)=\operatorname{Res}_{\lambda=0}\left(\partial A^{+} / \partial \lambda \wedge A_{-}\right)-d \operatorname{Res}_{\lambda=0}\left(\chi_{0}, A_{-}\right)
$$

and the result follows at once. 
Corollary 3.12. Let $V, \hat{V}$ be harmonic bundles with $\hat{V}$ a dressing transform of $V$. Then the harmonic map energy densities of $V$ and $\hat{V}$ differ by an exact 1-form.

So far, just as in Section 3.1, our analysis could be applied mutatis mutandis to $k$-perturbed harmonic maps into any symmetric space but now we specialize to the case of particular interest to us: we suppose that $V$ is the central sphere congruence of a constrained Willmore surface $\Lambda$ with multiplier $q$. In this case, Theorem 3.8 yields a 2-perturbed harmonic $\hat{V}$ and we are going to show that $\hat{V}$ is also the central sphere congruence of a constrained Willmore surface $\hat{\Lambda}$.

Our candidates for a new constrained Willmore surface and corresponding multiplier are

$$
\begin{aligned}
& \hat{\Lambda}=r(0) \Lambda^{1,0} \cap r(\infty) \Lambda^{0,1} \\
& \hat{q}=\hat{A}_{2}+\hat{A}_{-2}=\operatorname{Ad}_{r(\infty)} q^{1,0}+\operatorname{Ad}_{r(0)} q^{0,1}
\end{aligned}
$$

where the last identity is (3.10c). Since $\overline{r(0) \Lambda^{1,0}}=r(\infty) \Lambda^{0,1}$, Lemma $3.7(3)$ assures us that $\hat{\Lambda}$ is a real line subbundle of $\underline{\mathbb{C}}^{n+2}$.

We now have the main result of this section:

Theorem 3.13. Let $\left(V, d^{\lambda}\right)$ be the 2-perturbed harmonic central sphere congruence of a constrained Willmore surface $\Lambda$ with multiplier $q$. Let $\left(\hat{V}, \hat{d}^{\lambda}\right)$ be the dressing transform of $\left(V, d^{\lambda}\right)$ by $r$ and define $\hat{\Lambda}, \hat{q}$ by (3.11).

Then $\hat{\Lambda}$ is conformal and a constrained Willmore surface with multiplier $\hat{q}$ and central sphere congruence $\hat{V}$ on the open set where it immerses.

We therefore extend the terminology of Definition 3.5 and say that $\hat{\Lambda}$ is the dressing transform of $\Lambda$ by $r$.

Proof. There are three things to prove here. For conformality, we must show that $\hat{\Lambda}^{1,0}$ is isotropic. To see that $\hat{\Lambda}$ is constrained Willmore with multiplier $\hat{q}$, we need to show that $\hat{q}^{1,0} \in \Omega^{1,0}\left(\wedge^{2} \hat{\Lambda}^{0,1}\right)$ so that $\hat{q}$ gives rise to a holomorphic quadratic differential and that $\mathcal{N}_{\hat{V}}^{1,0} \hat{\Lambda}^{0,1}=0$ so that $\hat{V}$ is the central sphere congruence of $\hat{\Lambda}$. Theorem 2.6 then establishes the conclusion.

For all this, we prove that $\hat{\Lambda}^{0,1}=r(\infty) \Lambda^{0,1}$, whence, by reality, $\hat{\Lambda}^{1,0}=$ $r(0) \Lambda^{1,0}$, and $\mathcal{N}_{\hat{V}}^{0,1} r(0) \Lambda^{1,0}=0$. It is convenient to prove the second of these 
assertions first. From (3.10d), we have

$$
\mathcal{N}_{\hat{V}}^{0,1}=\operatorname{Ad}_{r(0)}\left(\mathcal{N}_{V}^{0,1}+\left[\chi_{0}(0), q^{0,1}\right]\right),
$$

so that we need to establish that

$$
\left(\mathcal{N}_{V}^{0,1}+\left[\chi_{0}(0), q^{0,1}\right]\right) \Lambda^{1.0}=0
$$

However, $\mathcal{N}_{V}^{0,1} \Lambda^{1,0}$ vanishes, since $V$ is the central sphere congruence of $\Lambda$, leaving us with the $\left[\chi_{0}(0), q^{0,1}\right]$ term. However, differentiating $(3.6)$ at $\lambda=0$ shows that $\chi_{0}(0)$ anti-commutes with $\rho_{V}$ and so takes values in $V \wedge V^{\perp}$. On the other hand, $q^{0,1}$ takes values in $\wedge^{2} \Lambda^{1,0}$ so that their bracket lies in $\Lambda^{1,0} \wedge V^{\perp}$ and so annihilates $\Lambda^{1,0}$ as required. Similarly, $\mathcal{N}_{\hat{V}}^{1,0} r(\infty) \Lambda^{0,1}=0$ and, in particular, $\mathcal{N}_{\hat{V}} \hat{\Lambda}$ vanishes.

Again $\hat{q}^{0,1} r(0) \Lambda^{1,0}=r(0) q^{0,1} \Lambda^{1,0}=0$ and, similarly, $\hat{q}^{1,0} r(\infty) \Lambda^{0,1}$ vanishes. In particular, $\hat{q} \hat{\Lambda}=0$.

It follows that the operators $d^{0,1}, \mathcal{D}_{\hat{V}}^{0,1}$ and $\left(\mathcal{D}_{\hat{V}}-\hat{q}\right)^{0,1}$ all coincide on $\Gamma \hat{\Lambda}$. However, (3.10a) reads

$$
\left(\mathcal{D}_{\hat{V}}-\hat{q}\right)^{0,1}=r(\infty) \cdot\left(\mathcal{D}_{V}-q\right)^{0,1}
$$

and $\Lambda^{0,1}$ is $\left(\mathcal{D}_{V}-q\right)^{0,1}$-stable whence $r(\infty) \Lambda^{0,1}$ is $\left(\mathcal{D}_{\hat{V}}-\hat{q}\right)^{0,1}$-stable. It follows at once that $d^{0,1}: \Gamma \hat{\Lambda} \rightarrow \Omega^{0,1}\left(r(\infty) \Lambda^{0,1}\right)$ so that $\hat{\Lambda}^{0,1}=r(\infty) \Lambda^{0,1}$ and, similarly, $\hat{\Lambda}^{1,0}=r(0) \Lambda^{1,0}$, since $\hat{\Lambda}$ immerses.

We remark that $q$ vanishes exactly when $\hat{q}$ does so that our dressing transforms when applied to Willmore surfaces give Willmore surfaces once more.

Proposition 3.11 applies in the current setting:

Corollary 3.14. Let $\hat{\Lambda}$ be a dressing transform of a constrained Willmore surface $\Lambda$. Then the Willmore densities of $\Lambda$ and $\hat{\Lambda}$ differ by an exact 1 -form.

Proof. We know from Section 2.2 that the Willmore density of a surface coincides with the harmonic map energy $\frac{1}{2}\left(\mathcal{N}_{V} \circ J^{\Sigma} \wedge \mathcal{N}_{V}\right)$ of its central sphere congruence $V$. On the other hand, in the present situation, the energy $e(V)$ of definition 3.10 is given by

$$
e(V)=\frac{1}{2}\left(\mathcal{N}_{V} \circ J \Sigma \wedge \mathcal{N}_{V}\right)+\left(q \circ J^{\Sigma} \wedge q\right)=\frac{1}{2}\left(\mathcal{N}_{V} \circ J^{\Sigma} \wedge \mathcal{N}_{V}\right)
$$

since $q$ takes values in the isotropic bundle $\Lambda \wedge \Lambda^{\perp}$. 
We conclude this analysis by showing that the holomorphic quadratic differentials that are the Lagrange multipliers for $\Lambda$ and $\hat{\Lambda}$ coincide. First, we extend the scope of (2.3):

Lemma 3.15. Let $\Lambda$ be a conformal immersion with central sphere congruence $V$. Let $q \in \Omega^{1}\left(\Lambda \wedge \Lambda^{(1)}\right.$ corresponding to a holomorphic quadratic differential $Q$ via (2.3). Then, for any $\tau \in \Gamma \Lambda^{1,0}$, we have

$$
Q^{0,2} \tau=q^{0,1}\left(\mathcal{D}_{V}^{0,1} \tau\right)
$$

Proof. Choose $Z \in T^{1,0} \Sigma$ and $\sigma \in \Gamma \Lambda$ with $\left(d_{Z} \sigma, d_{\bar{Z}} \sigma\right)=1$. Since $\Lambda \wedge \Lambda^{1,0}=$ $\wedge^{2} \Lambda^{1,0}$ has rank $1, q_{\bar{Z}}$ is a multiple of $\sigma \wedge d_{Z} \sigma$ and (2.3) quickly yields

$$
q_{\bar{Z}}=-Q(\bar{Z}, \bar{Z}) \sigma \wedge d_{Z} \sigma
$$

The right-hand side of (3.13) is tensorial in $\tau$ so that, in view of (2.3), it suffices to check (3.13) with $\tau=d_{Z} \sigma$. But

$$
\begin{aligned}
\left(\sigma \wedge d_{Z} \sigma\right)\left(\mathcal{D}_{V}\right)_{\bar{Z}} d_{Z} \sigma & =\left(\sigma,\left(\mathcal{D}_{V}\right)_{\bar{Z}} d_{Z} \sigma\right) d_{Z} \sigma-\left(d_{Z} \sigma,\left(\mathcal{D}_{V}\right)_{\bar{Z}} d_{Z} \sigma\right) \sigma \\
& =-\left(d_{\bar{Z}} \sigma, d_{Z} \sigma\right) d_{Z} \sigma+\frac{1}{2} d_{\bar{Z}}\left(d_{Z} \sigma, d_{Z} \sigma\right) \\
& =-d_{Z} \sigma
\end{aligned}
$$

and the result follows at once from (3.14).

Proposition 3.16. Let $\Lambda$ be a constrained Willmore surface and $\hat{\Lambda}$ the dressing transform of $\Lambda$ by $r$. Then $Q_{\hat{\Lambda}}=Q_{\Lambda}$.

Proof. From Lemma 3.15, for any section $\hat{\tau}$ of $\hat{\Lambda}^{1,0}$, we have

$$
Q_{\hat{\Lambda}}^{0,2} \hat{\tau}=\hat{q}^{0,1}\left(\mathcal{D}_{\hat{V}}^{0,1} \hat{\tau}\right)=\hat{q}^{0,1}\left(\left(\mathcal{D}_{\hat{V}}-\hat{q}\right)^{0,1} \hat{\tau}\right)
$$

We write $\hat{\tau}=\operatorname{Ad}_{r(0)} \tau$, for $\tau \in \Gamma \Lambda^{1,0}$, and apply (3.10) to see that

$$
\begin{aligned}
\hat{q}^{0,1}\left(\left(\mathcal{D}_{\hat{V}}-\hat{q}\right)^{0,1} \hat{\tau}\right)= & r(0) q^{0,1}\left(\left(\mathcal{D}_{V}-q\right)^{0,1} \tau\right) \\
& +\hat{q}^{0,1}\left(\frac{1}{2} \frac{\partial^{2}}{\partial \lambda^{2}}{ }_{\mid \lambda=0} \operatorname{Ad}_{r(\lambda)}\left(q^{0,1}+\lambda \mathcal{N}_{V}^{0,1}\right) \hat{\tau}\right) \\
= & Q_{\Lambda}^{0,2} \hat{\tau}+\hat{q}^{0,1}\left(\frac{1}{2} \frac{\partial^{2}}{\partial \lambda^{2}}{ }_{\mid \lambda=0} \operatorname{Ad}_{r(\lambda)}\left(q^{0,1}+\lambda \mathcal{N}_{V}^{0,1}\right) \hat{\tau}\right),
\end{aligned}
$$

so that the issue is to show that the last term vanishes. 
For this, note that

$$
\frac{\partial^{2}}{\partial \lambda^{2}}{ }_{\mid \lambda=0} \operatorname{Ad}_{r(\lambda)}\left(q^{0,1}+\lambda \mathcal{N}_{V}^{0,1}\right)=\operatorname{Ad}_{r(0)}\left(\left(\partial / \partial \lambda+\operatorname{ad}_{0}\right)^{2}\left(q^{0,1}+\lambda \mathcal{N}_{V}^{0,1}\right)_{\lambda=0}\right)
$$

so that it suffices to show that $C:=\left(\partial / \partial \lambda+\operatorname{ad} \chi_{0}\right)^{2}\left(q^{0,1}+\lambda \mathcal{N}_{V}^{0,1}\right)_{\lambda=0}$ preserves $\Lambda^{1,0}$. We may write (3.15) as

$$
\operatorname{Ad}_{r(0)} C=\frac{\partial^{2}}{\partial \lambda^{2}}{ }_{\mid \lambda=0} g(\lambda)
$$

where $g(\lambda)=\operatorname{Ad}_{r(\lambda)}\left(q^{0,1}+\lambda \mathcal{N}_{V}^{0,1}\right)$. From (3.6), we have $\operatorname{Ad}_{\rho_{\hat{V}}} g(\lambda)=g(-\lambda)$ and, differentiating this twice, we learn that $C$ commutes with $\rho_{V}$ and so preserves $V$. Since $\Lambda^{1,0}$ is maximal isotropic in $V$, we are reduced to showing that $\left(C \tau_{1}, \tau_{2}\right)$ vanishes for all $\tau_{1}, \tau_{2} \in \Lambda^{1,0}$. Now set $B_{\lambda}=(\partial / \partial \lambda+$ $\left.\operatorname{ad} \chi_{0}\right)\left(q^{0,1}+\lambda \mathcal{N}_{V}^{0,1}\right)$ so that $C=\left(\partial / \partial \lambda+\operatorname{ad} \chi_{0}\right)_{\mid \lambda=0} B_{\lambda}$. We observe that

$$
B_{0}=\mathcal{N}_{V}^{0,1}+\left[\chi_{0}(0), q^{0,1}\right]
$$

which, from (3.2), annihilates $\Lambda^{1,0}$. We compute:

$$
\begin{aligned}
\left(C \tau_{1}, \tau_{2}\right) & =\left(\partial / \partial \lambda_{\mid \lambda=0} B_{\lambda} \tau_{1}, \tau_{2}\right)+\left(\left[\chi_{0}(0), B_{0}\right] \tau_{1}, \tau_{2}\right) \\
& =\left(\partial / \partial \lambda_{\mid \lambda=0} B_{\lambda} \tau_{1}, \tau_{2}\right)-\left(B_{0} \tau_{1}, \chi_{0}(0) \tau_{2}\right)+\left(\chi_{0}(0) \tau_{1}, B_{0} \tau_{2}\right) \\
& =\partial / \partial \lambda_{\mid \lambda=0}\left(B_{\lambda} \tau_{1}, \tau_{2}\right) .
\end{aligned}
$$

However, $B_{\lambda}=\left(\partial / \partial \lambda+\operatorname{ad} \chi_{0}\right) A_{\lambda}$ for $A_{\lambda}=q^{0,1}+\lambda \mathcal{N}_{V}^{0,1}$ which last annihilates $\Lambda^{1,0}$, for all $\lambda$, so that repeating the last computation with $B_{\lambda}$ gives

$$
\left(B_{\lambda} \tau_{1}, \tau_{2}\right)=\partial / \partial \lambda\left(A_{\lambda} \tau_{1}, \tau_{2}\right)
$$

which vanishes identically.

\subsection{Bäcklund transformation}

Let us now remedy a lack in the analysis of the last section by providing examples of dressing gauges. For this, we follow Terng-Uhlenbeck [38] and contemplate dressing by simple factors.

Here is the basic building block of the construction: for $L^{+}, L^{-}$null line subbundles of $\underline{\mathbb{C}}^{n+2}$ which are complementary in the sense that $L_{x}^{+}$and $L_{x}^{-}$ 
are not orthogonal, for all $x \in \Sigma$, define $\Gamma_{L^{-}}^{L^{+}}: \mathbb{C}^{\times} \rightarrow \Gamma\left(\mathrm{O}\left(\underline{\mathbb{C}}^{n+2}\right)\right)$ by

$$
\Gamma_{L^{-}}^{L^{+}}(\lambda)= \begin{cases}\lambda & \text { on } L^{+} \\ 1 & \text { on }\left(L^{+} \oplus L^{-}\right)^{\perp} \\ \lambda^{-1} & \text { on } L^{-}\end{cases}
$$

The key property enjoyed by these gauge transformations is that $\operatorname{Ad} \Gamma_{L^{-}}^{L^{+}}(\lambda)$ is semisimple with eigenvalues $\lambda, 1$ and $\lambda^{-1}$ only.

Our simple factors will be constructed by precomposing these gauge transformations with a linear fractional transformation of $\lambda$ so the following simple lemma will be important for us:

Lemma 3.17. Let $\lambda \mapsto d^{\lambda}, \lambda \in \mathbb{C}^{\times}$, be a family of metric connections on $\underline{\mathbb{C}}^{n+2}$, holomorphic in $\lambda$ on an open subset of $\mathbb{P}^{1}$. Let $\alpha, \beta \in \mathbb{P}^{1}$ and $\psi_{\beta}^{\alpha}$ : $\mathbb{P}^{1} \rightarrow \mathbb{P}^{1}$ a linear fractional transformation with a zero at $\alpha$ and a pole at $\beta$. If $d^{\lambda}$ is holomorphic at $\alpha$, then the gauged family of connections

$$
\Gamma_{L^{-}}^{L^{+}}\left(\psi_{\beta}^{\alpha}(\lambda)\right) \cdot d^{\lambda}
$$

extend holomorphically across the singularity at $\alpha$ if and only if $L^{+}$is $d^{\alpha}$ parallel. Similarly, if $d^{\lambda}$ is holomorphic at $\beta$, the connections extend holomorphically at $\beta$ if and only if $L^{-}$is $d^{\beta}$-parallel.

Proof. Write $W=\left(L^{+} \oplus L^{-}\right)^{\perp}$, so that we have a decomposition $\underline{\mathbb{C}}^{n+2}=$ $L^{-} \oplus W \oplus L^{+}$. We have a corresponding decomposition of $d^{\alpha}$ :

$$
d^{\alpha}=D+\beta^{+}+\beta^{-}
$$

where $D$ preserves $L^{ \pm}$and $\beta^{ \pm} \in \Omega^{1}\left(L^{ \pm} \wedge W\right)$. We note that $L^{ \pm} \wedge W$ are the $\lambda^{ \pm 1}$-eigenbundles of $\operatorname{Ad} \Gamma_{L^{-}}^{L^{+}}(\lambda)$.

Now write $d^{\lambda}=d^{\alpha}+(\lambda-\alpha) B(\lambda)$ (we assume that $\alpha \neq \infty$, otherwise we work with $1 / \lambda)$. Then, with $\Gamma(\lambda)=\Gamma_{L^{-}}^{L^{+}}(\psi(\lambda))$

$$
\Gamma(\lambda) \cdot d^{\lambda}=\Gamma(\lambda) \cdot d^{\alpha}+(\lambda-\alpha) \operatorname{Ad}_{\Gamma(\lambda)} B(\lambda) .
$$

The second term is holomorphic near $\alpha$ since $\operatorname{Ad}_{\Gamma(\lambda)}$ introduces at most a simple pole at $\alpha$. As for the first term,

$$
\begin{aligned}
\Gamma(\lambda) \cdot d^{\alpha} & =\Gamma(\lambda) \cdot D+\operatorname{Ad}_{\Gamma(\lambda)}\left(\beta^{+}+\beta^{-}\right) \\
& =D+\psi(\lambda) \beta^{+}+\psi(\lambda)^{-1} \beta^{-},
\end{aligned}
$$


which is holomorphic near $\alpha$ if and only if $\beta^{-}$vanishes. However, this is the case if and only if $L^{+}$is $d^{\alpha}$-parallel.

The case at $\beta$ now follows after noting that $\Gamma_{L^{-}}^{L^{+}}\left(\psi_{\beta}^{\alpha}(\lambda)\right)=\Gamma_{L^{+}}^{L^{-}}\left(1 / \psi_{\beta}^{\alpha}(\lambda)\right)$ to which the first case applies.

Simple factors have only two poles so, in order to take care of both the twisting and reality conditions ${ }^{2}$ that Definition 3.5 imposes, we shall need a product of two simple factors (cf. [25, 27, 29]) and have recourse to a Bianchi permutability result to account for the non-commutativity of that product. For this and related results, the following lemma will be useful.

Lemma 3.18. Let $\ell^{+}, \ell^{-}$and $\hat{\ell}^{+}, \hat{\ell}^{-}$be two pairs of complementary null lines in $\mathbb{C}^{n+2}$. Let $\psi_{\beta}^{\alpha}: \mathbb{P}^{1} \rightarrow \mathbb{P}^{1}$ be a linear fractional transformation with zero at $\alpha$ and pole at $\beta$. Finally, let $E: \lambda \mapsto E(\lambda) \in \mathrm{O}\left(\mathbb{C}^{n+2}\right)$ be holomorphic near $\alpha$ and $\beta$. Then

$$
\lambda \mapsto \Gamma_{\hat{\ell}_{-}}^{\hat{\ell}^{+}}\left(\psi_{\beta}^{\alpha}(\lambda)\right) E(\lambda)\left(\Gamma_{\ell_{-}}^{\ell^{+}}\left(\psi_{\beta}^{\alpha}(\lambda)\right)\right)^{-1}
$$

is holomorphic at $\alpha$ if and only if $E(\alpha) \ell^{+}=\hat{\ell}^{+}$and holomorphic at $\beta$ if and only if $E(\beta) \ell^{-}=\hat{\ell}^{-}$.

Proof. Consider first the case where $\psi_{\beta}^{\alpha}(\lambda)=\lambda$. Here, holomorphicity of (3.16) at 0 is precisely the statement of [8, Lemma 4.10] while holomorphicity at $\infty$ follows by swapping the roles of $\ell^{ \pm}$and replacing $\lambda$ with $1 / \lambda$.

The case of arbitrary $\alpha, \beta$ can now be reduced to the first case by precomposing (3.16) with $\left(\psi_{\beta}^{\alpha}\right)^{-1}$.

Remark 3.19. Lemma 3.18 can be viewed as a discrete analogue of

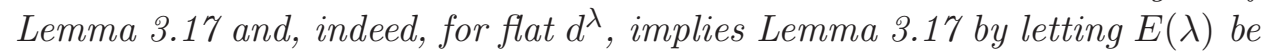
a gauge transform relating $d^{\lambda}$ and $d$.

With all this in hand, let $V$ be a non-degenerate subbundle of $\underline{\mathbb{C}}^{n+2}$, $\alpha \in \mathbb{C}^{\times}$and $L$ a null, line subbundle such that $L$ and $\rho_{V} L$ are complementary on an open set. From these data, define gauge transformations $p_{\alpha, L}^{V}(\lambda)$ on

\footnotetext{
${ }^{2}$ These conditions mean that if $\alpha$ is a pole, so is $-\alpha$ and $\pm 1 / \bar{\alpha}$.
} 
that open set by

$$
p_{\alpha, L}^{V}(\lambda)=\Gamma_{\rho_{V} L}^{L}\left(\frac{(1+\alpha)(\lambda-\alpha)}{(1-\alpha)(\lambda+\alpha)}\right)
$$

We observe:

$$
\begin{gathered}
p_{\alpha, L}^{V}(1)=1, \\
p_{\alpha, L}^{V}(-\lambda) \circ \rho_{V} \circ p_{\alpha, L}^{V}(\lambda)^{-1}=\rho_{V^{\prime}},
\end{gathered}
$$

where $V^{\prime}=p_{\alpha, L}^{V}(0) V=p_{\alpha, L}^{V}(\infty) V$,

$$
p_{\alpha, L}^{V}\left(\lambda^{*}\right)=\overline{p_{\alpha^{*}, \bar{L}}^{\bar{V}}(\lambda)}
$$

Moreover, $\lambda \mapsto p_{\alpha, L}^{V}(\lambda)$ is holomorphic on $\mathbb{P}^{1} \backslash\{ \pm \alpha\}$. We have

$$
\left(p_{\alpha, L}^{V}(0)\right)^{-1} p_{\alpha, L}^{V}(\infty)=\Gamma_{\rho_{V} L}^{L}(-1),
$$

the restriction of which to $V$ is reflection across $\left(L \oplus \rho_{V} L\right)^{\perp} \cap V$ which has codimension 1 in $V$ (otherwise $L=\rho_{V} L$ ) so that

$$
\operatorname{det}\left(\Gamma_{\rho_{V} L}^{L}(-1)\right)=-1
$$

We now have the main result of this section:

Theorem 3.20. Let $\left(V, d^{\lambda}\right)$ be k-perturbed harmonic, $\alpha \in \mathbb{C}^{\times} \backslash S^{1}$ and $L$ a $d^{\alpha}$-parallel null line subbundle with $L, \rho_{V} L$ complementary. Moreover, set $L^{\prime}=p_{\alpha, L}^{V}\left(\alpha^{*}\right) \bar{L}$ and $V^{\prime}=p_{\alpha, L}^{V}(0) V$. Assume also that $L^{\prime}, \rho_{V^{\prime}} L^{\prime}$ are complementary. Then $r:=p_{\alpha^{*}, L^{\prime}}^{V^{\prime}} p_{\alpha, L}^{V}$ is a dressing gauge for $\left(V, d^{\lambda}\right)$.

Thus, Theorem 3.8 applies with $r$ so defined to give a dressing transform of $V$ which, by Theorem 3.13, induces a transformation of constrained Willmore surfaces when $V$ is the central sphere congruence of such a surface.

Proof. First $r$ is rational in $\lambda$ on $\mathbb{P}^{1}$ and holomorphic on $\mathbb{P}^{1} \backslash\left\{ \pm \alpha, \pm \alpha^{*}\right\}$.

Since $d^{\lambda}$ is $\rho_{V}$-twisted, we see that $\rho_{V} L$ is $d^{-\alpha}$-parallel so that, by Lemma 3.17, $p_{\alpha, L}^{V}(\lambda) \cdot d^{\lambda}$ extends from $\mathbb{C}^{\times} \backslash\{ \pm \alpha\}$ to a holomorphic family of connections $\tilde{d}^{\lambda}$ on $\mathbb{C}^{\times}$. By the reality $(2.5 \mathrm{c})$ of $d^{\lambda}, \bar{L}$ is $d^{\alpha^{*}}$-parallel so that $L^{\prime}$ is $\tilde{d}^{\alpha^{*}}=p_{\alpha, L}^{V}\left(\alpha^{*}\right) \cdot d^{\alpha^{*}}$-parallel ${ }^{3}$. Moreover, we use $(3.17 \mathrm{~b})$ to see that $\tilde{d}^{\lambda}$ is $\rho_{V^{\prime}}$-twisted so that $\rho_{V^{\prime}} L^{\prime}$ is $\tilde{d}^{-\alpha^{*}}$-parallel. We therefore apply

\footnotetext{
${ }^{3}$ Here we use our hypothesis that $\alpha \notin S^{1}$.
} 
Lemma 3.17 again to see that $\hat{d}^{\lambda}=r(\lambda) \cdot d^{\lambda}=p_{\alpha^{*}, \hat{L}^{\prime}}^{V^{\prime}}(\lambda) \cdot \tilde{d}^{\lambda}$ is holomorphic on $\mathbb{C}^{\times}$with $\hat{d}^{1}=d$ since $r(1)=1$.

Two applications of $(3.17 \mathrm{~b})$ show that

$$
\begin{aligned}
r(-\lambda) \circ \rho_{V} \circ r(\lambda)^{-1} & =p_{\alpha^{*}, L^{\prime}}^{V^{\prime}}(-\lambda) \circ\left(p_{\alpha, L}^{V}(-\lambda) \circ \rho_{V} \circ p_{\alpha, L}^{V}(\lambda)^{-1}\right) \circ p_{\alpha^{*}, L^{\prime}}^{V^{\prime}}(\lambda)^{-1} \\
& =p_{\alpha^{*}, L^{\prime}}^{V^{\prime}}(-\lambda) \circ \rho_{V^{\prime}} \circ p_{\alpha^{*}, L^{\prime}}^{V^{\prime}}(\lambda)^{-1}
\end{aligned}
$$

is independent of $\lambda$ so that $r$ satisfies (3.6).

Meanwhile, from $(3.17 \mathrm{~d})$, we have

$$
\begin{aligned}
r(0)^{-1} r(\infty) & =\left(p_{\alpha^{*}, L^{\prime}}^{V^{\prime}}(0) p_{\alpha, L}^{V}(0)\right)^{-1} p_{\alpha^{*}, L^{\prime}}^{V^{\prime}}(\infty) p_{\alpha, L}^{V}(\infty) \\
& =p_{\alpha, L}^{V}(0)^{-1} \Gamma_{\rho_{V^{\prime}} L^{\prime}}^{L^{\prime}}(-1) p_{\alpha, L}^{V}(\infty) \\
& =\Gamma_{\rho_{V} L^{\prime \prime}}^{L^{\prime \prime}}(-1) \Gamma_{\rho_{V} L}^{L}(-1)
\end{aligned}
$$

where $L^{\prime \prime}=p_{\alpha, L}^{V}(0)^{-1} L^{\prime}$. Equation (3.17e) now tells us that $\operatorname{det}\left(r(0)^{-1} r(\infty)_{\mid V}\right)=1$.

We are therefore left to deal with the reality condition (3.4). For this, it suffices to prove that $\lambda \mapsto \overline{r\left(\lambda^{*}\right)} r(\lambda)^{-1}$ is holomorphic on $\mathbb{P}^{1}$ and so independent of $\lambda$. The conclusion then follows since $r(1)=1$. To stop the decorations piling up, let us temporarily write $M=L^{\prime}, U=V^{\prime}$ and $\beta=\alpha^{*}$ so that, using $(3.17 \mathrm{c})$,

$$
\overline{r(1 / \bar{\lambda})} r(\lambda)^{-1}=p_{\alpha, \bar{M}}^{\bar{U}}(\lambda) p_{\beta, \bar{L}}^{V}(\lambda) p_{\alpha, L}^{V}(\lambda)^{-1} p_{\beta, M}^{U}(\lambda)^{-1}
$$

This is holomorphic in $\lambda$ on $\mathbb{P}^{1} \backslash\{ \pm \alpha, \pm \beta\}$. However, Lemma 3.18 tells us that $p_{\beta, \bar{L}}^{V} p_{\alpha, L}^{V}{ }^{-1} p_{\beta, M}^{U}{ }^{-1}$ is holomorphic at $\beta$ since $\bar{L}=p_{\alpha, L}^{V}(\beta)^{-1} M$ and holomorphic at $-\beta$ since $\rho_{V} \bar{L}=p_{\alpha, L}^{V}(-\beta)^{-1} \rho_{V^{\prime}} M$. Since $p_{\alpha, \bar{M}}^{\bar{U}}$ is also holomorphic at $\pm \beta$, we see that $\overline{r\left(\lambda^{*}\right)} r(\lambda)^{-1}$ is holomorphic there also.

A similar argument with $p_{\alpha, \bar{M}}^{\bar{U}} p_{\beta, \bar{L}}^{V} p_{\alpha, L}^{V}{ }^{-1}$ using $\bar{M}=p_{\beta, \bar{L}}^{V}(\alpha) L$ establishes holomorphicity at $\pm \alpha$ and we are done.

Remark 3.21. To construct bundles L that satisfy the hypotheses of Theorem 3.20 it suffices to choose an initial condition $L_{x}$, some $x \in \Sigma$, that satisfies the (open) complementarity conditions and extend to an open set by parallel translation since $d^{\alpha}$ is flat. We observe that the complementarity conditions are non-empty since they are satisfied by real, null $L_{x}$ transverse to $V_{x}$ and $V_{x}^{\perp}$. Indeed, in this case, $L_{x}^{\prime}=p_{\alpha, L_{x}}^{V}\left(\alpha^{*}\right) L_{x}=L_{x}$ and $\rho_{V_{x}^{\prime}} L_{x}^{\prime}=\rho_{V} L_{x}$ since $L_{x}$ is an eigenspace of $p_{\alpha, L_{x}}^{V}(\lambda)$, for all $\lambda$. 
By analogy with the Bianchi-Bäcklund transform of constant Gauss curvature surfaces (cf. [29]), we make the following

Definition 3.22. The dressing transform by the dressing gauge $r$ of Theorem 3.20 is the Bäcklund transform with parameters $\alpha, L$.

Remark 3.23. In the classical literature, Bäcklund transforms are specified by a choice of spectral parameter $\alpha$ and an initial condition which locates the transformed surface in space at a base-point $x \in \Sigma$. In our setting, this last amounts to choosing the fibre $L_{x}$ from which our bundle $L$ can be recovered by parallel translation with respect to the flat connection $d^{\alpha}$.

\subsection{Bianchi permutability for Bäcklund transforms}

Our terminology in Definition 3.22 may be justified by the fact that there is a Bianchi permutability theorem available for our Bäcklund transform.

Let us recall in outline the statement of Bianchi permutability: we start with a $k$-perturbed harmonic $\left(V, d^{\lambda}\right)$ and construct two Bäcklund transforms $\left(V_{1}, d_{1}^{\lambda}\right),\left(V_{2}, d_{2}^{\lambda}\right)$ with parameters $\alpha_{1}, L_{1}$ and $\alpha_{2}, L_{2}$, respectively. The theorem then asserts the existence of a fourth $k$-perturbed harmonic $\left(V_{12}, d_{12}\right)$ which is simultaneously a Bäcklund transform of $V_{1}$ with parameter $\alpha_{2}$ and of $V_{2}$ with parameter $\alpha_{1}$.

This amounts to finding the right line bundles $L_{2}^{1}$ and $L_{1}^{2}$ with $L_{2}^{1} d_{1}^{\alpha_{2}}$ parallel and $L_{1}^{2} d_{2}^{\alpha_{1}}$-parallel. However, we have natural candidates close at hand: by construction, we have gauge transformations $r_{1}, r_{2}$ with $r_{j}(\lambda) \cdot d^{\lambda}=$ $d_{j}^{\lambda}$ and so

$$
\begin{aligned}
& L_{2}^{1}:=r_{1}\left(\alpha_{2}\right) L_{2}, \\
& L_{1}^{2}:=r_{2}\left(\alpha_{1}\right) L_{1}
\end{aligned}
$$

fit the bill. It remains to verify that the Bäcklund transformations of $V_{j}$ with parameters $\alpha_{k}, L_{k}^{j}, j \neq k \in\{1,2\}$, coincide and it is to this that we now turn.

We begin with an effort to keep the notation under control: as already indicated, we denote by $r_{j}$ the dressing gauge that implements the Bäcklund transform of $\left(V, d^{\lambda}\right)$ with parameters $\alpha_{j}, L_{j}$. Similarly, let $r_{j}^{k}$ be the dressing gauge that implements the Bäcklund transform of $V_{k}$ with parameters $\alpha_{j}, L_{j}^{k}$. Thus, for example,

$$
r_{1}(\lambda)=p_{\alpha_{1}^{*}, L_{1}^{\prime}}^{*} p_{\alpha_{1}, L_{1}}^{V}
$$


where $*$ denotes a bundle (in fact $p_{\alpha_{1}, L_{1}}^{V}(0) V$ ) that we will not have to keep track of. We have $d_{1}^{\lambda}=r_{1}(\lambda) \cdot d^{\lambda}$, off a divisor, $V_{1}=r_{1}(0) V=r_{1}(\infty) V$, and, in case that $V$ is the central sphere congruence of a constrained Willmore $\Lambda, \Lambda_{1}=r_{1}(0) \Lambda^{1,0} \cap r_{1}(\infty) \Lambda^{0,1}$.

The key fact is contained in the following lemma:

Lemma 3.24. Suppose that $\alpha_{2} \notin\left\{ \pm \alpha_{1}, \pm \alpha_{1}^{*}\right\}$. Then

$$
r_{2}^{1} r_{1}=r_{1}^{2} r_{2}
$$

Proof. We shall argue as in the proof of Theorem 3.20 (itself in part a permutability theorem for $p$ 's) and show that $\Pi:=r_{1}^{2} r_{2}\left(r_{1}\right)^{-1}\left(r_{2}^{1}\right)^{-1}$ is holomorphic on $\mathbb{P}^{1}$ and so constant. Then (3.20) follows by evaluating at $\lambda=1$.

First we consider holomorphicity at $\alpha_{1}$ : we know that $r_{2}^{1}$ is holomorphic and invertible at $\alpha_{1}$ so the issue is with $r_{1}^{2} r_{2}\left(r_{1}\right)^{-1}$ which reads

$$
p_{\alpha_{1}^{*}, \hat{L}_{1}^{2}}^{*} p_{\alpha_{1}, L_{1}^{2}}^{V_{2}} r_{2} p_{\alpha_{1}, L_{1}}^{V}{ }^{-1} p_{\alpha_{1}^{*}, \hat{L}_{1}}^{*}{ }^{-1}
$$

Here, the outer $p^{*}$-terms are holomorphic at $\alpha_{1}$ (recall that $\alpha_{1} \notin S^{2}$ !) and what is left is holomorphic thanks to Lemma 3.18 and (3.19b). Thus, our product $\Pi$ is holomorphic and $\mathrm{O}\left(\underline{\mathbb{C}}^{n+2}\right)$-valued at $\alpha_{1}$. The same argument, with the roles of $\alpha_{1}$ and $\alpha_{2}$ swopped shows that $r_{2}^{1} r_{1}\left(r_{2}\right)^{-1}$ and so $r_{2}\left(r_{1}\right)^{-1}\left(r_{2}^{1}\right)^{-1}$ is holomorphic and invertible at $\alpha_{2}$.

For the remaining potential singularities at $-\alpha_{j}, \pm \alpha_{j}^{*}$, we observe that, first, $\Pi$ is real: $\overline{\Pi(\lambda)}=\Pi\left(\lambda^{*}\right)$ and then that, with $\rho_{1}^{2}$ being the reflection across $\Pi_{1}^{2}(0) V_{2}$ and similarly for $\rho_{2}^{1}$, we have

$$
\rho_{2}^{1} \Pi(\lambda)=\Pi(-\lambda) \rho_{1}^{2} .
$$

Consequently, holomorphicity at $-\alpha_{j}, \pm \alpha_{j}^{*}$ follows from that at $\alpha_{j}$ and $\Pi$ is holomorphic on $\mathbb{P}^{1}$ as required.

With this in hand, the permutability theorem follows quickly: with $r$ the common value in (3.20), set $V_{12}=r(0) V=r(\infty) V, d_{12}^{\lambda}=r(\lambda) \cdot d^{\lambda}$ and, in case we are working with a constrained Willmore surface $\Lambda_{12}=r(0) \Lambda^{1,0} \cap$ $r(\infty) \Lambda^{0,1}$. Then

$$
\begin{aligned}
& V_{12}=r_{2}^{1} r_{1}(0) V=r_{2}^{1}(0) V_{1} \\
& d_{12}^{\lambda}=r_{2}^{1}(\lambda) \cdot\left(r_{1}(\lambda) \cdot d^{\lambda}\right)=r_{2}^{1}(\lambda) \cdot d_{1}^{\lambda}, \\
& \Lambda_{12}=\left(r_{2}^{1} r_{1}(0) \Lambda^{1,0}\right) \cap\left(r_{2}^{1} r_{1}(\infty) \Lambda^{0,1}\right)=r_{2}^{1}(0) \Lambda_{1}^{1,0} \cap r_{2}^{1}(\infty) \Lambda_{1}^{0,1}
\end{aligned}
$$


so that $\left(V_{12}, d_{12}^{\lambda}\right)$ (respectively $\left.\Lambda_{12}\right)$ is the Bäcklund transform of $\left(V_{1}, d_{1}^{\lambda}\right)$ (respectively $\Lambda_{1}$ ) with parameters $\alpha_{2}, L_{2}^{1}$. Similarly, these data are the Bäcklund transform of $\left(V_{2}, d_{2}^{\lambda}\right)$ (respectively $\Lambda_{2}$ ) with parameters $\alpha_{1}, L_{1}^{2}$. To summarize:

Theorem 3.25. Let $\left(V, d^{\lambda}\right)$ be k-perturbed harmonic and $\alpha_{1}, \alpha_{2} \in \mathbb{C}^{\times} \backslash S^{1}$ with $\alpha_{2} \notin\left\{ \pm \alpha_{1}, \pm \alpha_{1}^{*}\right\}$. Let $\left(V_{1}, d_{1}^{\lambda}\right),\left(V_{2}, d_{2}^{\lambda}\right)$ be the Bäcklund transforms of $\left(V, d^{\lambda}\right)$ with parameters $\alpha_{1}, L_{1}$ and $\alpha_{2}, L_{2}$ respectively. Then, with $L_{j}^{k}$ defined by (3.19), the Bäcklund transform of $\left(V_{1}, d_{1}^{\lambda}\right)$ with parameters $\alpha_{2}, L_{2}^{1}$ coincides with the Bäcklund transform of $\left(V_{2}, d_{2}^{\lambda}\right)$ with parameters $\alpha_{1}, L_{1}^{2}$.

Moreover, if $V$ is the central sphere congruence of a constrained Willmore surface $\Lambda$, the corresponding iterated transforms of $\Lambda$ coincide also.

\subsection{Spectral deformation versus Dressing transform}

We conclude our analysis by comparing the spectral and dressing transformations. In an appropriate sense, these commute. For this, begin with a $k$-perturbed harmonic map $\left(V, d^{\lambda}\right)$. Recall from Section 3.1 that, for $\mu \in$ $S^{1}$, we have $k$-perturbed harmonic maps $\left(V_{\mu}, d_{\mu}^{\lambda}\right)$ given by $V_{\mu}=\Phi_{\mu} V$ and $d_{\mu}^{\lambda}=\Phi_{\mu} \cdot d^{\lambda \mu}$ where $\Phi_{\mu} \in \Gamma \mathrm{O}\left(\underline{\mathbb{R}}^{n+1,1}\right)$ solves $\Phi_{\mu} \cdot d_{\mu}=d$. Now let $\left(\hat{V}, \hat{d}^{\lambda}\right)$ be a dressing transform of $\left(V, d^{\lambda}\right)$ via a dressing gauge $r$. Then $\left(\hat{V}, \hat{d}^{\lambda}\right)$ has a spectral deformation $\left(\hat{V}_{\mu}, \hat{d}_{\mu}^{\lambda}\right)$ given by $\hat{V}_{\mu}=\hat{\Phi}_{\mu} \hat{V}, \hat{d}_{\mu}^{\lambda}=\hat{\Phi}_{\mu} \cdot \hat{d}^{\lambda \mu}$, where $\hat{\Phi}_{\mu}$ solves $\hat{\Phi}_{\mu} \cdot \hat{d}^{\mu}=d$.

For $\lambda \in \operatorname{dom}(r) \backslash\{0, \infty\}$, we have $\hat{d}^{\lambda}=r(\lambda) \cdot d^{\lambda}$ so that, defining $r_{\mu}(\lambda):=$ $\hat{\Phi}_{\mu} r(\lambda \mu)\left(\Phi_{\mu}\right)^{-1}$, we have

$$
\begin{aligned}
& \hat{d}_{\mu}^{\lambda}=r_{\mu}(\lambda) \cdot d_{\mu}^{\lambda}, \quad \lambda \in \operatorname{dom}\left(r_{\mu}\right) \backslash\{0, \infty\}, \\
& \hat{V}_{\mu}=r_{\mu}(0) V_{\mu} .
\end{aligned}
$$

It is now a simple matter to check that $r_{\mu}$ is a dressing gauge for $V_{\mu}$ and we conclude that $\hat{V}_{\mu}$ is a dressing transform of $V_{\mu}$. Thus:

Proposition 3.26. Let $\left(\hat{V}, \hat{d}^{\lambda}\right)$ be a dressing transform of a $k$-perturbed harmonic $\left(V, d^{\lambda}\right)$. For $\mu \in S^{1}$, the spectral deformation $\left(\hat{V}_{\mu}, \hat{d}_{\mu}^{\lambda}\right)$ of $\left(\hat{V}, \hat{d}^{\lambda}\right)$ is a dressing transform of the spectral deformation $\left(V_{\mu}, d_{\mu}^{\lambda}\right)$ of $\left(V, d^{\lambda}\right)$.

In case that $\left(\hat{V}, \hat{d}^{\lambda}\right)$ is a Bäcklund transform of $\left(V, d^{\lambda}\right)$, we can do a little better. First observe that, in this case, $\mu \in \operatorname{dom}(r) \backslash\{0, \infty\}$ so that $\hat{d}^{\mu}=r(\mu) \cdot d^{\mu}=\left(r(\mu) \Phi_{\mu}^{-1}\right) \cdot d$ and we may therefore take $\hat{\Phi}_{\mu}=\Phi_{\mu} r(\mu)^{-1}$. Further, suppose that the Bäcklund transformation of $\left(V, d^{\lambda}\right)$ has parameters 
$\alpha, L$ so that $L$ is a $d^{\alpha}$-parallel, null line subbundle. Then $\Phi_{\mu} L$ is $d_{\mu}^{\mu / \alpha}$-parallel which prompts the following result:

Proposition 3.27. Let $\left(\hat{V}, \hat{d}^{\lambda}\right)$ be the Bäcklund transform of $\left(V, d^{\lambda}\right)$ with parameters $\alpha, L$. For $\mu \in S^{1}$, the spectral deformation $\left(\hat{V}_{\mu}, \hat{d}_{\mu}^{\lambda}\right)$ is the Bäcklund transform with parameters $\alpha / \mu, \Phi_{\mu} L$ of the spectral deformation $\left(V_{\mu}, d_{\mu}^{\lambda}\right)$.

Proof. In this case, we have $r(\lambda)=p_{\alpha^{*}, L^{\prime}}^{V^{\prime}}(\lambda) p_{\alpha, L}^{V}(\lambda)$. We set $L_{\mu}=\Phi_{\mu} L$. Then, in view of the discussion above, our result amounts to the identity

$$
\Phi_{\mu} r(\mu)^{-1} r(\lambda \mu) \Phi_{\mu}^{-1}=p_{\alpha^{*} / \mu, L_{\mu}^{\prime}}^{V^{\prime}}(\lambda) p_{\alpha / \mu, L_{\mu}}^{V_{\mu}}(\lambda)
$$

For this, we use the, by now familiar, permutability argument. Rearranging all terms onto the left-hand side yields a product which is holomorphic in $\lambda$ except possibly at $\pm \alpha / \mu$ and $\pm \alpha^{*} / \mu$. The part with possible singularities at $\pm \alpha / \mu$ is

$$
\Gamma_{\rho_{V} L}^{L}\left(\frac{\lambda \mu-\alpha}{\lambda \mu+\alpha}\right) \Phi_{\mu}^{-1}\left(\Gamma_{\rho_{V_{\mu}} L_{\mu}}^{L_{\mu}}\left(\frac{\lambda-\alpha / \mu}{\lambda+\alpha / \mu}\right)\right)^{-1}
$$

which is readily seen to reduce to $\Phi_{\mu}^{-1}$ and so is independent of $\lambda$. The reality condition now establishes holomorphicity at $\pm \alpha^{*} / \mu$ so that the whole product is independent of $\lambda$ and we are done.

In particular, we see that any Bäcklund transform can be obtained as a combination of spectral deformations and a Bäcklund transform with $\alpha \in \mathbb{R}$.

\section{Codimension 2}

Conformal surface geometry in $S^{4}$ has a distinctive flavour of its own. This manifests itself in various ways such as a well-developed twistor theory $[6,17$, $20]$ and the quaternionic formalism of the Berlin school $[2,10,19]$. However, for us, the key feature of this setting is that the space of oriented 2-spheres is a complex manifold (in fact, a pseudo-Hermitian symmetric space). We will show that this structure allows us both to simplify the preceding theory and to relate it to a construction of Willmore surfaces in [10].

\subsection{Additional structure in codimension 2}

We consider maps of the Riemann surface $\Sigma$ into the space $\tilde{G}_{3,1}\left(\mathbb{R}^{5,1}\right)$ of oriented 2-spheres in $S^{4}$, or, equivalently, oriented bundles $V$ of $(3,1)$-planes in 
$\mathbb{C}^{6}$. Note that if $V$ is the central sphere congruence of a conformal immersion $\Lambda$, then $\sigma \wedge d_{X} \sigma \wedge d_{J^{\Sigma} X} \sigma \wedge \Delta \sigma, X \in T \Sigma, \sigma \in \Gamma \Lambda$, gives an orientation of $V$ which is independent of choices.

In this setting, $V^{\perp}$ is a real bundle of oriented (2,0)-planes and so splits as a sum of complex conjugate, $\mathcal{D}^{V}$-parallel, null line subbundles:

$$
V^{\perp}=V_{+}^{\perp} \oplus V_{-}^{\perp}
$$

As a consequence, we have a family of $\mathcal{D}^{V}$-parallel gauge transformations $\tau_{V}(\lambda):=\Gamma_{V_{-}^{\perp}}^{V_{+}^{\perp}}(\lambda)$ and, in particular, an almost complex structure $J^{V}$ on $V \wedge V^{\perp}$ given by $J^{V}=\tau_{V}(\sqrt{-1})_{\mid V \wedge V^{\perp}}$. We may therefore split $\mathcal{N}_{V}$ into its holomorphic and anti-holomorphic parts:

$$
\mathcal{N}_{V}=\mathcal{A}_{V}+\mathcal{Q}_{V}
$$

with $\mathcal{A}_{V} \circ J^{\Sigma}=J^{V} \circ \mathcal{A}_{V}$ and $\mathcal{Q}_{V} \circ J^{\Sigma}=-J^{V} \circ \mathcal{Q}_{V}$. Thus $\mathcal{A}_{V}^{1,0} \in \Omega^{1,0}(V \wedge$ $\left.V_{+}^{\perp}\right)$ while $\mathcal{Q}_{V}^{1,0} \in \Omega^{1,0}\left(V \wedge V_{-}^{\perp}\right)$.

We note the case where $\mathcal{A}=0$ (respectively, $\mathcal{Q}=0$ ): this means that $V$ is a (anti-)holomorphic map into the space of oriented 2 -spheres or, equivalently, $V_{+}^{\perp}$ (respectively, $\left.V_{-}^{\perp}\right)$ is a holomorphic subbundle of $\underline{\mathbb{C}}^{6}$. If, in addition, $V$ is the central sphere congruence of a conformal immersion $\Lambda$, then this condition obtains if and only if $\Lambda$ is twistor holomorphic, that is, $\Lambda$ has a holomorphic twistor lift to $\mathbb{C} P^{3}[10$, Section 8.1 and Lemma 22]. Such $\Lambda$ are, of course, Willmore surfaces.

\subsection{Untwisted family of flat connections}

Let $\left(V, d^{\lambda}\right)$ be a $k$-perturbed harmonic map into $\tilde{G}_{3,1}\left(\mathbb{R}^{5,1}\right)$. Since $\lambda \mapsto \tau_{V}(\lambda)$ is a group homomorphism $\mathbb{C}^{\times} \rightarrow \mathrm{O}\left(\underline{\mathbb{C}}^{6}\right)$ and $\tau_{V}(-1)=\rho_{V}$, we readily conclude from (2.5b) that

$$
\tau_{V}(\lambda) \cdot d^{\lambda}=\tau_{V}(-\lambda) \cdot d^{-\lambda}
$$

Thus there is a second family of connections $\nabla^{\mu}, \mu \in \mathbb{C}^{\times}$, defined by

$$
\nabla^{\lambda^{2}}=\tau_{V}(\lambda) \cdot d^{\lambda}
$$

for $\lambda \in \mathbb{C}^{\times}$. 
Proposition 4.1. Let $\left(V, d^{\lambda}\right)$ be a k-perturbed harmonic map into $\tilde{G}_{3,1}\left(\mathbb{R}^{5,1}\right)$ with $d^{\lambda}=\sum_{|i| \leq k} A_{k}$. Define $\nabla^{\mu}, \mu \in \mathbb{C}^{\times}$, by (4.3). Then:

$$
\begin{aligned}
\nabla^{1} & =d & & \text { (normalization) } \\
\nabla^{\mu^{*}} & =\overline{\nabla^{\mu}} & & \text { (reality) }
\end{aligned}
$$

and $\mu \mapsto\left(\nabla^{\mu}\right)^{1,0}$ is holomorphic on $\mathbb{C}$ with a pole of order $l$ at $\infty$ (whence, by (4.4b), $\mu \mapsto\left(\nabla^{\mu}\right)^{0,1}$ is holomorphic on $\mathbb{C}^{\times} \cup\{\infty\}$ with a pole of order $l$ at 0$)$. Here

$$
l= \begin{cases}(k-1) / 2 & \text { if } k \text { is odd and } A_{k} V_{-}^{\perp}=0 \\ k / 2 & \text { if } k \text { is even; } \\ (k+1) / 2 & \text { otherwise. }\end{cases}
$$

Moreover $V_{-}^{\perp}$ is $\left(\nabla^{0}\right)^{1,0}$-stable (whence $V_{+}^{\perp}$ is $\left(\nabla^{\infty}\right)^{0,1}$-stable).

Proof. We have $\tau_{V}(1)=1$ and $\tau_{V}\left(\lambda^{*}\right)=\overline{\tau_{V}(\lambda)}$ which, together with (2.5), readily yields $(4.4)$.

For $A \in \Omega^{1}\left(\wedge^{2} \underline{\mathbb{C}}^{6}\right)$, decompose $A=A^{+}+A^{0}+A^{-}$according to the eigenspace decomposition of $\operatorname{Ad}_{\tau_{V}(\lambda)}$ :

$$
\wedge^{2} \underline{\mathbb{C}}^{6}=\left(V \wedge V_{+}^{\perp}\right) \oplus\left(\wedge^{2} V \oplus \wedge^{2} V^{\perp}\right) \oplus\left(V \wedge V_{-}^{\perp}\right)
$$

Then

$$
\left(\nabla^{\lambda^{2}}\right)^{1,0}=\left(A_{0}+A_{1}^{-}\right)+\lambda^{2}\left(A_{1}^{+}+A_{2}+A_{3}^{-}\right)+\cdots,
$$

which establishes the holomorphicity of $\mu \mapsto\left(\nabla^{\mu}\right)^{1,0}$ on $\mathbb{C}$. Moreover, as $A_{0}$ preserves $V_{ \pm}^{\perp}$ while $A_{1}^{-}$annihilates $V_{-}^{\perp}$, we conclude that $V_{-}^{\perp}$ is $\left(\nabla^{0}\right)^{1,0_{-}}$ stable.

Finally, we contemplate the leading term in $\lambda$ of $\left(\nabla^{\lambda^{2}}\right)^{1,0}$ : if $k$ is even, this is $\lambda^{k}\left(A_{k}+A_{k-1}^{+}\right)$, while, for odd $k$, it is either $\lambda^{k+1} A_{k}^{+}$, if $A_{k}^{+} \neq 0$, or $\lambda^{k-1}\left(A_{k-1}+A_{k}^{-}\right)$when $A_{k}^{+}=0$, or, equivalently, $A_{k} V_{-}^{\perp}=0$.

Thus, these connections share many properties of the family $d^{\lambda}$ but are not $\rho_{V}$-twisted and have poles of half the order. In view of the first property, we call $\nabla^{\mu}$ the untwisted family of connections associated to $\left(V, d^{\lambda}\right)$.

We can reverse this line of argument and start from an untwisted family of flat connections:

Theorem 4.2. Let $V$ be a bundle of $(3,1)$-planes in $\underline{\mathbb{R}}^{5,1}$ and $\nabla^{\mu}=$ $\sum_{|j| \leq l} \mu^{j} B_{j}, \mu \in \mathbb{C}^{\times}$, a family of flat connections satisfying the conclusions of Proposition 4.1 . 
Define connections $d^{\lambda}$ by

$$
d^{\lambda}=\tau_{V}(\lambda)^{-1} \cdot \nabla^{\lambda^{2}}
$$

$\lambda \in \mathbb{C}^{\times}$. Then $d^{\lambda}$ satisfies the conditions of Definition 2.5, so that $\left(V, d^{\lambda}\right)$ is a $k$-perturbed harmonic bundle where

$$
k= \begin{cases}2 l-1 & \text { if } B_{l}^{-}=B_{l}^{0}=0 \\ 2 l & \text { if } B_{l}^{-}=0 \text { but } B_{l}^{0} \neq 0 \\ 2 l+1 & \text { otherwise }\end{cases}
$$

Proof. It is clear that, with $d^{\lambda}$ defined by (4.6), we have $d^{1}=d$ and that the reality condition $(2.5 \mathrm{c})$ holds. Moreover, we readily compute:

$$
d^{-\lambda}=\tau_{V}(-\lambda)^{-1} \cdot \nabla^{\lambda^{2}}=\left(\rho_{V} \tau_{V}(\lambda)^{-1}\right) \cdot \nabla^{\lambda^{2}}=\rho_{V} \cdot d^{\lambda}
$$

so that the twisting condition (2.5b) holds also.

As for the dependence in $\lambda$ of $d^{\lambda}$, it is clear that $\lambda \mapsto d^{\lambda}$ is holomorphic on $\mathbb{C}^{\times}$while the argument of Lemma 3.17 can be easily adapted to treat the family of partial connections $\lambda \mapsto\left(\nabla^{\lambda^{2}}\right)^{1,0}$ and we deduce that $\left(d^{\lambda}\right)^{1,0}$ is holomorphic at 0 since $V_{-}^{\perp}$ is $\left(\nabla^{0}\right)^{1,0}$-stable. Finally, the leading terms in $\lambda$ of $d^{\lambda}$ are

$$
\lambda^{2 k+1} B_{l}^{-}+\lambda^{2 k} B_{l}^{0}+\lambda^{2 k-1}\left(B_{l}^{+}+B_{l-1}^{-}\right)+\cdots
$$

from which (4.7) follows at once.

We conclude that the families $d^{\lambda}$ and $\nabla^{\mu}$ carry the same information and therefore refer to a $k$-perturbed harmonic bundle in codimension 2 as either $\left(V, d^{\lambda}\right)$ or $\left(V, \nabla^{\mu}\right)$, where $d^{\lambda}$ and $\nabla^{\mu}$ are related via (4.3) and (4.6).

We list some examples:

(1) $\nabla^{\mu} \equiv d$ satisfies the conditions of Proposition 4.1 if and only if $V_{+}^{\perp}$ is holomorphic. This is the case $l=0$ of Theorem 4.2.

(2) $V$ is harmonic if and only if $\nabla^{\mu}=d+(\mu-1) \mathcal{A}^{1,0}+\left(\mu^{-1}-1\right) \mathcal{A}^{0,1}$ is flat, for all $\mu \in \mathbb{C}^{\times}$. A similar result is true for maps into any (pseudo-) Hermitian symmetric space.

(3) Let $V$ be the central sphere congruence of $\Lambda$. Then $\Lambda$ is constrained Willmore with multiplier $q$ if and only if $\nabla^{\mu}=d+(\mu-1)(q+\mathcal{A})^{1,0}+$ $\left(\mu^{-1}-1\right)(q+\mathcal{A})^{0,1}$ is flat, for all $\mu \in \mathbb{C}^{\times}[2]$. This is the case $l=1$ of Theorem 4.2 with $B_{1}^{-}=0$ and the additional constraint that $B_{1}^{0}$ take values in $\Lambda \wedge \Lambda^{(1)}$. 


\subsection{Untwisted dressing gauges}

The untwisted family of connections has a simpler algebraic structure. It is therefore unsurprising that we may dress such families with algebraically simpler gauge transformations:

Definition 4.3. Let $\left(V, \nabla^{\mu}\right)$ be a $k$-perturbed harmonic bundle in $\underline{\mathbb{C}}^{6}$. An untwisted dressing gauge for $\left(V, \nabla^{\mu}\right)$ is a family of gauge transformations $\mu \mapsto R(\mu) \in \Gamma \mathrm{O}\left(\underline{\mathbb{C}}^{6}\right)$ which is holomorphic in $\mu$ near $0, \infty \in \mathbb{P}^{1}$ and has the following properties:

(a) For all $\mu \in \operatorname{dom}(R)$,

$$
R\left(\mu^{*}\right)=\overline{R(\mu)} \quad \text { (reality). }
$$

(b) The connections $R(\mu) \cdot \nabla^{\mu}$ extend from $\operatorname{dom}(R) \backslash\{0, \infty\}$ to a holomorphic family of connections $\hat{\nabla}^{\mu}$ on $\mathbb{C}^{\times}$with $\hat{\nabla}^{1}=d$.

(c) The line bundles $R(0) V_{-}^{\perp}$ and $R(\infty) V_{+}^{\perp}$ are complementary.

For such an $R$, set $\hat{V}=\left(R(0) V_{-}^{\perp} \oplus R(\infty) V_{+}^{\perp}\right)^{\perp}$, oriented so that

$$
\hat{V}_{-}^{\perp}=R(0) V_{-}^{\perp}, \quad \hat{V}_{+}^{\perp}=R(\infty) V_{+}^{\perp},
$$

and call $\left(\hat{V}, \hat{\nabla}^{\mu}\right)$ the untwisted dressing transform of $\left(V, \nabla^{\mu}\right)$ by $R$.

We note that $\hat{V}$ is a bundle of $(3,1)$-planes since $\hat{V}^{\perp}$ is a sum of complex conjugate null lines.

It is our contention that $\left(\hat{V}, \hat{\nabla}^{\mu}\right)$ is again $k$-perturbed harmonic. This will follow at once from the next proposition which reduces the situation to the twisted setup of Section 3.2.

Proposition 4.4. Let $\left(\hat{V}, \hat{\nabla}^{\mu}\right)$ be the untwisted dressing transform of $\left(V, \nabla^{\mu}\right)$ by $R$. Set

$$
r(\lambda)=\tau_{\hat{V}}(\lambda)^{-1} \circ R\left(\lambda^{2}\right) \circ \tau_{V}(\lambda)
$$

Then $r$ is a dressing gauge for $\left(V, d^{\lambda}\right)$ and, with $\hat{d}^{\lambda}=\tau_{\hat{V}}(\lambda)^{-1} \cdot \hat{\nabla}^{\lambda^{2}},\left(\hat{V}, \hat{d}^{\lambda}\right)$ is the dressing transform of $\left(V, d^{\lambda}\right)$ by $r$.

In particular, $\left(V, d^{\lambda}\right)$ is k-perturbed harmonic by Theorem 3.8.

Proof. We check that $r$ defined by (4.10) satisfies the conditions of Definition 3.5. For this, first note that Lemma 3.18 along with (4.9), shows that 
$\lambda \mapsto r(\lambda)$ is holomorphic near 0 and $\infty$. Now, for item (1) of Definition 3.5, a short calculation gives

$$
r(-\lambda) \circ \rho_{V} \circ r(\lambda)^{-1}=\rho_{\hat{V}},
$$

which is certainly independent of $\lambda$. Item (2) is immediate from the corresponding reality conditions on $R, \tau_{V}, \tau_{\hat{V}}$. In particular, evaluating at $\lambda=0$ gives $r(\infty)=\overline{r(0)}$. Again, with $d^{\lambda}=\tau_{V}(\lambda)^{-1} \cdot \nabla^{\lambda^{2}}$, we have, from item (b) of Definition 4.3, that $\hat{d}^{\lambda}=r(\lambda) \cdot d^{\lambda}$ on $\operatorname{dom}(r) \backslash\{0, \infty\}$. However, it is clear from its definition that $\lambda \mapsto \hat{d}^{\lambda}$ is holomorphic on $\mathbb{C}^{\times}$and that $\hat{d}^{1}=d$. This settles item (3). Finally, for item (4), evaluate (4.11) at $\lambda=0, \infty$ to deduce that $\hat{V}=r(0) V=r(\infty) V$. We have already noted that $\hat{V}$ is a bundle of $(3,1)$-planes and now Lemma 3.6(2) together with $r(\infty)=\overline{r(0)}$ immediately yields (3.5).

We therefore conclude that $r$ is indeed a dressing gauge for $\left(V, d^{\lambda}\right)$. Moreover, since $r(\lambda) \cdot d^{\lambda}=\hat{d}^{\lambda}$, for $\lambda \in \operatorname{dom}(r) \backslash\{0, \infty\}$, and $\hat{V}=r(0) V$, we see that $\left(\hat{V}, \hat{d}^{\lambda}\right)$ is the dressing transform of $\left(V, d^{\lambda}\right)$ by $r$.

With an eye to dressing constrained Willmore surfaces, we compute $r(0)$ and $r(\infty)$ :

Lemma 4.5. With $V, \hat{V}$ and $r, R$ as in Proposition 4.4, define projections $\pi_{i}, \hat{\pi}_{i}$ by

$$
\begin{aligned}
& \tau_{V}(\lambda)=\lambda \pi_{1}+\pi_{0}+\lambda^{-1} \pi_{-1}, \\
& \tau_{\hat{V}}(\lambda)=\lambda \hat{\pi}_{1}+\hat{\pi}_{0}+\lambda^{-1} \hat{\pi}_{-1} .
\end{aligned}
$$

Then:

$$
r(0)=\sum_{|i| \leq 1} \hat{\pi}_{i} R(0) \pi_{i} \quad r(\infty)=\sum_{|i| \leq 1} \hat{\pi}_{i} R(\infty) \pi_{i}
$$

Proof. We have already seen that $r(0) V=r(\infty) V=\hat{V}$ but more is true: rearrange (4.10) to give

$$
R\left(\lambda^{2}\right)=\tau_{\hat{V}}(\lambda) \circ r(\lambda) \circ \tau_{V}(\lambda)^{-1}
$$

so that the holomorphicity of $\lambda \mapsto R\left(\lambda^{2}\right)$ near 0 and $\infty$, along with Lemma 3.18, tells us that $r(0) V_{+}^{\perp}=\hat{V}_{+}^{\perp}$ and $r(\infty) V_{-}^{\perp}=\hat{V}_{-}^{\perp}$. Moreover, since $r(0)$ is an 
isometry and $V_{-}^{\perp}$ is maximal isotropic in $V^{\perp}$, we have that $r(0) V_{-}^{\perp}$ is maximal isotropic in $\hat{V}^{\perp}$ and so must be $\hat{V}_{-}^{\perp}$. Thus

$$
r(0)=\sum_{|i| \leq 1} \hat{\pi}_{i} r(0) \pi_{i}
$$

and similarly for $r(\infty)$.

However,

$$
\sum_{|i| \leq 1} \hat{\pi}_{i} r(\lambda) \pi_{i}=\sum_{|i| \leq 1} \hat{\pi}_{i} \tau_{\hat{V}}(\lambda)^{-1} R\left(\lambda^{2}\right) \tau_{V}(\lambda) \pi_{i}=\sum_{|i| \leq 1} \hat{\pi}_{i} R\left(\lambda^{2}\right) \pi_{i}
$$

and evaluating this last at $\lambda=0, \infty$ establishes (4.12).

Putting all this together with Theorem 3.13 yields:

Theorem 4.6. Let $\left(V, \nabla^{\mu}\right)$ be the 2-perturbed harmonic central sphere congruence of a constrained Willmore surface $\Lambda$ with multiplier $q$. Let $\left(\hat{V}, \hat{\nabla}^{\mu}\right)$ be the untwisted dressing transform of $\left(V, \nabla^{\mu}\right)$ by $R$.

Define:

$$
\begin{aligned}
\hat{\Lambda} & =\left(\pi_{\hat{V}} R(0) \Lambda^{1,0}\right) \cap\left(\pi_{\hat{V}} R(\infty) \Lambda^{0,1}\right) \\
\hat{q} & =\operatorname{Ad}_{\pi_{\hat{V}} R(\infty)} q^{1,0}+\operatorname{Ad}_{\pi_{\hat{V}} R(0)} q^{0,1}
\end{aligned}
$$

Then $\hat{\Lambda}$ is a constrained Willmore surface with multiplier $\hat{q}$ and central sphere congruence $\hat{V}$ on the open set where it immerses.

Definition 4.7. We say that $\hat{\Lambda}$ is the untwisted dressing transform of $\Lambda$ by $R$.

We shall see below in Proposition 4.12 and Corollary 4.13 that a quaternionic formalism provides cleaner formulae in which the projections $\pi_{\hat{V}}$ do not intervene.

Remark 4.8. Which dressing gauges $r$ arise from untwisted dressing gauges $R$ via (4.10)? Lemma 4.5 provides a necessary condition: $r(0) V_{ \pm}^{\perp}=\hat{V}_{ \pm}^{\perp}$ and $r(\infty) V_{ \pm}^{\perp}=\hat{V}_{ \pm}^{\perp}$. It is not difficult to see that this condition is also sufficient by arguing as in Proposition 4.4 .

Any dressing gauge has $r(0) V^{\perp}=\hat{V}^{\perp}$ and $r(0) V_{ \pm}^{\perp}$ maximal isotropic in $\hat{V}^{\perp}$, so that the remaining possibility is that $r(0) V_{ \pm}^{\perp}=\hat{V}_{\mp}^{\perp}$ and $r(\infty) V_{ \pm}^{\perp}=\hat{V}_{\mp}^{\perp}$. This amounts to switching the orientation of $\hat{V}^{\perp}$ or, equivalently, replacing 
$\tau_{\hat{V}}$ by its inverse. We leave it as an exercise to the interested reader to work out the analogue of our analysis in this case.

\subsection{Untwisted simple factors}

The lack of $\rho_{V}$-twisting allows the possibility of untwisted dressing gauges with just two poles so that, in codimension 2, we may dress by simple factors. For this, let $\left(V, d^{\lambda}\right)=\left(V, \nabla^{\mu}\right)$ be a $k$-perturbed harmonic bundle. For $\nu \in \mathbb{C}^{\times} \backslash S^{1}$, let $M$ be a null, line subbundle such that $M$ and $\bar{M}$ are complementary on an open set ${ }^{4}$. Use this data to define gauge transformations $P_{\nu, M}(\mu)$ on that set by

$$
P_{\nu, M}(\mu)=\Gamma \frac{M}{M}\left(\frac{\left(1-\nu^{*}\right)(\mu-\nu)}{(1-\nu)\left(\mu-\nu^{*}\right)}\right) .
$$

We have:

$$
\begin{gathered}
P_{\nu, M}(1)=1, \\
P_{\nu, M}\left(\mu^{*}\right)=\overline{P_{\nu, M}(\mu)},
\end{gathered}
$$

and that $\mu \mapsto P_{\nu, M}(\mu)$ is rational on $\mathbb{P}^{1}$ and holomorphic on $\mathbb{P}^{1} \backslash\left\{\nu, \nu^{*}\right\}$.

Theorem 4.9. Let $\left(V, \nabla^{\mu}\right)$ be a k-perturbed harmonic bundle in $\underline{\mathbb{C}}^{6}, \nu \in$ $\mathbb{C}^{\times} \backslash S^{1}$ and $M$ a $\nabla^{\nu}$-parallel null line subbundle of $\underline{\mathbb{C}}^{6}$. Assume that $M, \bar{M}$ are complementary and then that $P_{\nu, M}(\infty) V_{+}^{\perp}$ and $P_{\nu, M}(0) V_{-}^{\perp}$ are complementary. Then $P_{\nu, M}$ is an untwisted dressing gauge for $\left(V, \nabla^{\mu}\right)$.

Proof. We have already seen that $\mu \mapsto P_{\nu, M}(\mu)$ is holomorphic on $\mathbb{P}^{1} \backslash\left\{\nu, \nu^{*}\right\}$ and so, in particular, near 0 and $\infty$. Moreover, (4.14b) is precisely the reality condition (4.8) of Definition 4.3.

We have that $M$ is $\nabla^{\nu}$-parallel, when, thanks to $(4.14 \mathrm{~b}), \bar{M}$ is $\nabla^{\nu^{*}}$ parallel. It is now immediate from Lemma 3.17 that $\mu \mapsto P_{\nu, M}(\mu) \cdot \nabla^{\mu}$ extends holomorphically to $\mathbb{C}^{\times}$settling Definition $4.3(\mathrm{~b})$.

Finally, Definition 4.3(c) holds by hypothesis.

In fact, the dressing transformation induced by these simple factor dressing gauges are generically Bäcklund transforms as in Section 3.3. Indeed, if $M$ is $\nabla^{\nu}$-parallel and $\alpha= \pm \sqrt{\nu}$, then (4.6) tells us that $L:=\tau_{V}(\alpha)^{-1} M$ is $d^{\alpha}$-parallel. Thus $\alpha, L$ are parameters for a Bäcklund transform of $\left(V, d^{\lambda}\right)$ which turns out to have the same effect as dressing by $P_{\nu, M}$. In more detail:

\footnotetext{
${ }^{4}$ This is the open set on which $M$ is not real.
} 
Proposition 4.10. Let $\left(V, \nabla^{\mu}\right)$ be a k-perturbed harmonic bundle in $\underline{\mathbb{C}}^{6}$, $\nu \in \mathbb{C}^{\times} \backslash S^{1}$ and $M a \nabla^{\nu}$-parallel null line subbundle satisfying the hypotheses of Theorem 4.9. Let $\left(\hat{V}, \hat{\nabla}^{\mu}\right)$ be the dressing transform of $\left(V, \nabla^{\mu}\right)$ by $P_{\nu, M}$.

Let $\alpha= \pm \sqrt{\nu}$ and set $L=\tau_{V}(\alpha)^{-1} M$. Assume that $L$ satisfies the hypotheses of Theorem 3.20: thus $L, \rho_{V} L$ are complementary and, with $L^{\prime}=p_{\alpha, L}^{V}\left(\alpha^{*}\right) \bar{L}$ and $V^{\prime}=p_{\alpha, L}^{V}(0) V, L^{\prime}, \rho_{V^{\prime}} L^{\prime}$ are also complementary.

Then

$$
p_{\alpha^{*}, L^{\prime}}^{V^{\prime}}(\lambda) p_{\alpha, L}^{V}(\lambda)=\tau_{\hat{V}}(\lambda)^{-1} P_{\nu, M}\left(\lambda^{2}\right) \tau_{V}(\lambda)
$$

for all $\lambda$.

Thus, Proposition 4.4 tell us that $\left(\hat{V}, \hat{d}^{\lambda}\right)$ is the Bäcklund transform of $\left(V, d^{\lambda}\right)$ with parameters $\alpha, L$.

Proof. Set

$$
\begin{aligned}
& L(\lambda):=p_{\alpha^{*}, L^{\prime}}^{V^{\prime}}(\lambda) p_{\alpha, L}^{V}(\lambda), \\
& R(\lambda):=\tau_{\hat{V}}(\lambda)^{-1} P_{\nu, M}\left(\lambda^{2}\right) \tau_{V}(\lambda) .
\end{aligned}
$$

We follow what should now be a familiar strategy: we use Lemma 3.18 to see that $\lambda \mapsto R(\lambda) L(\lambda)^{-1}$ is holomorphic on $\mathbb{P}^{1}$ and so constant.

Both $L(\lambda)$ and $R(\lambda)$ are holomorphic on $\mathbb{P}^{1} \backslash \pm \alpha, \pm \alpha^{*}, L(\lambda)$ by construction and $R(\lambda)$ by Proposition 4.4. Moreover, both $R, L$ satisfy the reality condition so it suffices to show holomorphicity of $R L^{-1}$ at $\pm \alpha$. Now the part of this product with poles at $\pm \alpha$ is

$$
\begin{aligned}
\Gamma \frac{M}{M}\left(\frac{\lambda^{2}-\alpha^{2}}{\lambda^{2}-1 / \bar{\alpha}^{2}}\right) \tau_{V}(\lambda) \Gamma_{\rho_{V} L}^{L}\left(\frac{\lambda-\alpha}{\lambda+\alpha}\right)^{-1} & =\Gamma \frac{M}{M}\left(\frac{\lambda+\alpha}{\lambda+\alpha^{*}}\right) \Gamma_{\frac{M}{M}}\left(\frac{\lambda-\alpha}{\lambda-\alpha^{*}}\right) \tau_{V}(\lambda) \Gamma_{\rho_{V} L}^{L}\left(\frac{\lambda-\alpha}{\lambda+\alpha}\right)^{-1} \\
& =\Gamma \frac{M}{M}\left(\frac{\lambda-\alpha}{\lambda-\alpha^{*}}\right) \Gamma \frac{M}{M}\left(\frac{\lambda+\alpha}{\lambda+\alpha^{*}}\right) \tau_{V}(\lambda) \Gamma_{L}^{\rho_{V} L}\left(\frac{\lambda+\alpha}{\lambda-\alpha}\right)^{-1} .
\end{aligned}
$$

We have $M=\tau_{V}(\alpha) L$ and this, together with Lemma 3.18 applied to the right hand side of (4.16a), gives holomorphicity at $\alpha$. Again, $\tau_{V}(-\alpha) \rho_{V} L=$ $\tau_{V}(\alpha) L=M$, so that Lemma 3.18 applies to (4.16b) to give holomorphicity at $-\alpha$.

\subsection{Quaternionic formalism}

The Klein correspondence offers another viewpoint on the conformal geometry of $S^{4}$ which has been heavily exploited by Pedit, Pinkall and their 
collaborators $[2,3,10,19]$. We rehearse the basics of this viewpoint with a view to extending the transformation theory of [10, Chapter 12] and relating it to our dressing transformations in Section 4.6.

We begin with a 2-dimensional quaternionic vector space which we view as $\mathbb{C}^{4}$ equipped with a quaternionic structure $j$ : thus $j: \mathbb{C}^{4} \rightarrow \mathbb{C}^{4}$ is antilinear and $j^{2}=-1$. Now fix $\operatorname{det} \in \wedge^{2}\left(\mathbb{C}^{4}\right)^{*}$ with

$$
j^{*} \operatorname{det}=\overline{\operatorname{det}}, \quad \operatorname{det}\left(v_{1} \wedge v_{2} \wedge j v_{1} \wedge j v_{2}\right)>0,
$$

when $v_{1} \wedge v_{2} \wedge j v_{1} \wedge j v_{2} \neq 0 v_{i} \in \mathbb{C}^{4}$. These data equip $\mathbb{C}^{6}:=\wedge^{2} \mathbb{C}^{4}$ with a real structure $\overline{v_{1} \wedge v_{2}}:=j v_{1} \wedge j v_{2}$ and an inner product $(\xi, \eta):=\operatorname{det}(\xi \wedge \eta)$ of signature $(5,1)$.

Set $\mathrm{SL}\left(\mathbb{H}^{2}\right)=\left\{g \in \mathrm{SL}\left(\mathbb{C}^{4}\right): j g=g j\right\}$. The action of $\mathrm{SL}\left(\mathbb{H}^{2}\right)$ on $\wedge^{2} \mathbb{C}^{4}$ induces a map $\mathrm{SL}\left(\mathbb{H}^{2}\right) \rightarrow \mathrm{SO}(5,1)$ which is a double cover. Differentiating gives an isomorphism of Lie algebras $\mathfrak{s l}\left(\mathbb{H}^{2}\right) \cong \mathfrak{o}\left(\mathbb{R}^{5,1}\right)$ where $\mathfrak{s l}\left(\mathbb{H}^{2}\right)=\{A \in$ $\left.\mathfrak{s l}\left(\mathbb{C}^{4}\right):[A, j]=0\right\}$.

The Klein correspondence identifies the Grassmannian of 2-planes in $\mathbb{C}^{4}$ with the quadric in $\mathbb{P}\left(\mathbb{C}^{6}\right)$ defined by our inner product: $W \mapsto \wedge^{2} W$. Clearly, $j$-stable 2-planes (thus one-dimensional quaternionic subspaces) are identified with points of $\mathbb{P}(\mathcal{L})=S^{4}$ yielding the celebrated isomorphism $\mathbb{H} P^{1} \cong S^{4}$.

Under this correspondence, oriented 2-spheres are identified with 2planes $S^{+} \leq \mathbb{C}^{4}$ which are not $j$-stable: for such an $S^{+}$, set $S^{-}=j S^{+}$and then $\mathbb{C}^{4}=S^{+} \oplus S^{-}$and the corresponding $(3,1)$-plane $V$ in $\mathbb{R}^{5,1}$ is given by:

$$
V=S^{+} \wedge S^{-}, \quad V_{ \pm}^{\perp}=\wedge^{2} S^{ \pm}
$$

Equivalently, such $S^{+}$correspond bijectively to $S \in \operatorname{SL}\left(\mathbb{H}^{2}\right)$ with $S^{2}=-1$ via $S= \pm i$ on $S^{ \pm}$.

Thus, a bundle $V$ of oriented $(3,1)$-planes amounts to $S \in \Gamma \mathrm{SL}\left(\underline{\mathbb{H}}^{2}\right)$ with $S^{2}=-1$ and $\pm i$-eigenbundles $S^{ \pm}$so that $V_{ \pm}^{\perp}=\wedge^{2} S^{ \pm}$. The corresponding decomposition $\underline{\mathbb{C}}^{4}=S^{+} \oplus S^{-1}$ induces a decomposition of the flat connection

$$
d=\mathcal{D}_{S}+\mathcal{N}_{S}
$$

with $\mathcal{D}_{S} S=0$ and $\mathcal{N}_{S}$ anti-commuting with $S:\left\{\mathcal{N}_{S}, S\right\}=0$. Using $V=$ $S^{+} \wedge S^{-}$, we readily see that $\mathcal{D}_{S}$ induces the connection $\mathcal{D}_{V}$ on $\underline{\mathbb{C}}^{6}=\wedge^{2} \underline{\mathbb{C}}^{4}$ and that $\mathcal{N}_{S}$ coincides with $\mathcal{N}_{V}$ under the isomorphism $\mathfrak{s l}\left(\mathbb{H}^{2}\right) \cong \mathfrak{o}(5,1)$. Again, the subspace $V \wedge V^{\perp}$ of $\mathfrak{o}(5,1)$ corresponds to $\left\{A \in \mathfrak{s l}\left(\mathbb{H}^{2}\right):\{A, S\}=\right.$ $0\}$ with the almost complex structure $J_{V}$ corresponding to post-composition 
with $S$. Thus the decomposition (4.1) of $\mathcal{N}_{V}$ is identified with the decomposition

$$
\mathcal{N}_{S}=\mathcal{A}_{S}+\mathcal{Q}_{S}
$$

of [10, Section 5.1] where

$$
\begin{aligned}
& * \mathcal{A}_{S}=S \mathcal{A}_{S}=-\mathcal{A}_{S} S, \\
& * \mathcal{Q}_{S}=-S \mathcal{Q}_{S}=\mathcal{Q}_{S} S .
\end{aligned}
$$

Here, and below, we follow [10] by setting $* \alpha=\alpha \circ J^{\Sigma}$, for $\alpha \in \Omega_{\Sigma}^{1}$. Thus our $*$ is minus that of Hodge.

We note the following simple consequences of this analysis [10, Equations (5.2) and (5.11)]:

$$
\begin{aligned}
d S & =2\left(* \mathcal{Q}_{S}-* \mathcal{A}_{S}\right), \\
0 & =\mathcal{A}_{S} \wedge \mathcal{Q}_{S}, \\
0 & =\mathcal{Q}_{S} \wedge \mathcal{A}_{S},
\end{aligned}
$$

where coefficients in the wedge products are multiplied using composition in $\operatorname{End}\left(\mathbb{C}^{4}\right)$.

Now let $\Lambda$ be a conformal immersion with oriented central sphere congruence $V$. Then $\Lambda$ corresponds to a quaternionic line subbundle of $\underline{\mathbb{C}}^{4}$, thus a $j$-stable, rank 2 bundle $L \leq \underline{\mathbb{C}}^{4}$ with $\wedge^{2} L=\Lambda$. To the central sphere congruence $V$ corresponds $S \in \Gamma \mathrm{SL}\left(\underline{\mathbb{H}}^{2}\right)$ characterized by the following conditions [10, Section 5.2, Theorem 2]:

$$
\begin{gathered}
S L=L, \quad d S L \leq T^{*} \Sigma \otimes L, \\
* \delta=S \delta=\delta S, \\
\mathcal{Q}_{S} L=0,
\end{gathered}
$$

where $\delta=\pi \circ d$ for $\pi: \underline{\mathbb{C}}^{4} \rightarrow \underline{\mathbb{C}}^{4} / L$ the projection away from $L$.

From (4.19a), we see that $L=L^{+} \oplus L^{-}$where $L^{ \pm}=L \cap S^{ \pm}$and $L^{-}=$ $j L^{+}$. Thus $\Lambda=L^{+} \wedge L^{-}$. We remark that $L^{ \pm}$, viewed as maps $\Sigma \rightarrow \mathbb{C} P^{3}$ are the two twistor lifts of $\Lambda$. Moreover, from $(4.19 \mathrm{~b})$, we readily compute that:

$$
\Lambda^{1,0}=S^{+} \wedge L^{-}, \quad \Lambda^{0,1}=L^{+} \wedge S^{-}
$$

We have therefore established a dictionary between subbundles of $\underline{\mathbb{C}}^{4}$ and $\underline{\mathbb{C}}^{6}=\wedge^{2} \underline{\mathbb{C}}^{4}$ which we shall use without further comment. Thus we can (and will!) talk of conformal immersions $L$ with central sphere congruence $S$. 
With a view to working with constrained Willmore surfaces in this formalism, we list the algebraic properties of a Lagrange multiplier $q$ when viewed as an $\mathfrak{s t}\left(\mathbb{H}^{2}\right)$-valued 1 -form.

Lemma 4.11. Let $\Lambda=\wedge^{2} L$ be a conformal immersion with central sphere congruence $S$. Let $q \in \Omega^{1} \otimes \mathfrak{o}(5,1)$. Then $q^{1,0}$ takes values in $\Lambda \wedge \Lambda^{0,1}$ if and only if, when viewed as a $\mathfrak{s l}\left(\mathbb{H}^{2}\right)$-valued 1 -form, we have

$$
\begin{gathered}
q \in \Omega^{1}\left(\operatorname{End}\left(\mathbb{H}^{2} / L, L\right)\right) \\
* q=S q=q S .
\end{gathered}
$$

Proof. Suppose that $q^{1,0}$ takes values in $\Lambda \wedge \Lambda^{0,1}$, or, equivalently, $q$ preserves $V$ and annihilates $V^{\perp}$ while $q^{1,0}$ additionally annihilates $\Lambda^{0,1}$. The first two of these imply that $q S=S q$ so that $q$ preserves $S^{ \pm}$. Moreover, $q V^{\perp}=0$ means that $q \wedge^{2} S^{ \pm}=0$, so that $\operatorname{trace}\left(q_{\mid S^{ \pm}}\right)=0$.

Let $Z \in T^{1,0} \Sigma, \sigma^{+} \in L^{+}$and $s^{-} \in S^{-}$. Then

$$
q_{Z}\left(\sigma^{+} \wedge s^{-}\right)=\left(q_{Z} \sigma^{+}\right) \wedge s^{-}+\sigma^{+} \wedge\left(q_{Z} s^{-}\right)=0
$$

It follows at once that $L^{+}$is an eigenspace of $q_{Z}$ with eigenvalue $\lambda$, say, and then that $q_{Z}=-\lambda$ on $S^{-}$. Since $q_{Z}$ is trace-free on $S^{-}$, we immediately get that $q_{Z}$ annihilates $S^{-}$(so that $* q=q S$ ) and also $L^{+}$and so $L$. Finally, choose $s^{+} \in S^{+}$so that $\sigma^{+} \wedge s^{+} \neq 0$. Then $0=q_{Z}\left(\sigma^{+} \wedge s^{+}\right)=$ $\sigma^{+} \wedge\left(q_{Z} s^{+}\right)$so that $q_{Z} S^{+} \leq L^{+}$. This establishes Equations (4.20). The converse is straightforward.

Let $R$ be a dressing gauge for a $k$-perturbed harmonic $\left(S, \nabla^{\mu}\right)$. We wish to describe its effect in quaternionic terms for which we need a little notation: for $g \in \mathrm{O}\left(\mathbb{C}^{6}\right)$, write $\widetilde{g}$ for a preimage of $g$ under the double covering $\mathrm{SL}\left(\mathbb{C}^{4}\right) \rightarrow \mathrm{O}\left(\mathbb{C}^{6}\right)$. Thus

$$
g(v \wedge w)=(\widetilde{g} v) \wedge(\widetilde{g} w)
$$

for $v, w \in \mathbb{C}^{4}$. Clearly $\widetilde{g}$ is determined up to sign by $g$ and has an unambiguous projective action on $\mathbb{C}^{4}$.

With this in hand, we have:

Proposition 4.12. Let $\left(S, \nabla^{\mu}\right)$ be k-perturbed harmonic in $\underline{\mathbb{C}}^{6}$. Let $R$ be an untwisted dressing gauge for $\left(S, \nabla^{\mu}\right)$ and $\left(\hat{S}, \hat{\nabla}^{\mu}\right)$ the untwisted dressing 
transform by $R$. Then

$$
\hat{S}^{+}=\widetilde{R}(\infty) S^{+} \quad \hat{S}^{-}=\widetilde{R}(0) S^{-} .
$$

Moreover, if $S$ is the central sphere congruence of a constrained Willmore surface $L$ with multiplier $q$, then the dressing transform $\hat{S}$ and its multiplier $\hat{q}$ are given by:

$$
\begin{aligned}
\hat{L}^{+} & =\widetilde{R}(\infty) L^{+} \quad \hat{L}^{-}=\widetilde{R}(0) S^{-} \\
\hat{q}_{\mid \hat{S}^{+}} & =\widetilde{R}(\infty) q \widetilde{R}(\infty)_{\mid \hat{S}^{+}}^{-1} \quad \hat{q}_{\mid \hat{S}^{-}}=\widetilde{R}(0) q \widetilde{R}(0)_{\mid \hat{S}^{-}}^{-1}
\end{aligned}
$$

Proof. First note that the right members of (4.21) follow at once from the left members because $R(0)=\bar{R}(\infty)$ so that we may take $\widetilde{R}(0)$ to be $j \circ$ $\widetilde{R}(\infty) \circ j^{-1}$.

For (4.21a), first note that (4.9) amounts to $\wedge^{2} \hat{S}^{+}=R(\infty) \wedge^{2} S^{+}$, which immediately yields $\hat{S}^{+}=\widetilde{R}(\infty) S^{+}$.

For the rest, let $\pi^{ \pm}, \hat{\pi}^{ \pm}$be the projections corresponding to the decompositions $\underline{\mathbb{C}}^{4}=S^{+} \oplus S^{-}$and $\underline{\mathbb{C}}^{4}=\hat{S}^{+} \oplus \hat{S}^{-}$. It is easy to deduce from Lemma 4.5 that

$$
\begin{aligned}
\widetilde{r}(\infty) & =\hat{\pi}^{+} \widetilde{R}(\infty) \pi^{+}+\hat{\pi}^{-} \widetilde{R}(\infty) \pi^{-}=\widetilde{R}(\infty) \pi^{+}+\hat{\pi}^{-} \widetilde{R}(\infty) \pi^{-} \\
\widetilde{r}(0) & =\hat{\pi}^{+} \widetilde{R}(0) \pi^{+}+\hat{\pi}^{-} \widetilde{R}(0) \pi^{-}=\hat{\pi}^{+} \widetilde{R}(0) \pi^{+}+\widetilde{R}(0) \pi^{-} .
\end{aligned}
$$

In particular,

$$
r(\infty) \Lambda^{0,1}=\left(\widetilde{R}(\infty) L^{+}\right) \wedge\left(\hat{\pi}^{-} \widetilde{R}(\infty) S^{-}\right)=\left(\widetilde{R}(\infty) L^{+}\right) \wedge \hat{S}^{-},
$$

where, for the last equality, we note that $\hat{\pi}^{-} \widetilde{R}(\infty)$ has kernel $S^{+}$and so injects (therefore surjects) when restricted to $S^{-}$. Complex conjugation now yields $r(0) \Lambda^{1,0}=\hat{S}^{+} \wedge \widetilde{R}(0) L^{-}$and then (3.11a) gives

$$
\hat{\Lambda}=\left(\hat{S}^{+} \wedge \widetilde{R}(0) L^{-}\right) \cap\left(\widetilde{R}(\infty) L^{+} \wedge \hat{S}^{-}\right)=\widetilde{R}(\infty) L^{+} \wedge \widetilde{R}(0) L^{-} .
$$

In particular, $\hat{L}^{+}=\widetilde{R}(\infty) L^{+}$and $\hat{L}^{-}=\widetilde{R}(0) L^{-}$settling (4.21b).

Finally, we consider $q$. From $(4.20 \mathrm{~b})$, we see that $q$ preserves $S^{ \pm}$while $q_{\mid S^{-}}^{1,0}=0$ and similarly for $\hat{q}$. On the other hand, from (3.11b), we have:

$$
\hat{q}^{1,0}=\operatorname{Ad}_{r(\infty)} q^{1,0}=\widetilde{r}(\infty) q^{1,0} \widetilde{r}(\infty)^{-1}
$$

Since $\widetilde{r}(\infty)_{\mid S^{+}}=\widetilde{R}(\infty)_{\mid S^{+}}$with image $\hat{S}^{+}$, this immediately yields

$$
\hat{q}_{\mid \hat{S}^{+}}^{1,0}=\widetilde{R}(\infty) q^{1,0} \widetilde{R}(\infty)_{\mid \hat{S}^{+}}^{-1} .
$$


On the other hand, both $\hat{q}^{0,1}$ and $\widetilde{R}(\infty) q^{0,1} \widetilde{R}(\infty)^{-1}$ vanish on $\hat{S}^{+}$and so (4.21c) is established.

We summarize the development in the following corollary:

Corollary 4.13. Let $R$ be an untwisted dressing gauge of a $k$-perturbed harmonic $\left(S, \nabla^{\mu}\right)$ in $\underline{\mathbb{C}}^{6}$ and define $T_{0} \in \Gamma \mathrm{GL}\left(\underline{\mathbb{H}}^{2}\right)$ by

$$
T_{0}=\widetilde{R}(\infty) \pi^{+}+\widetilde{R}(0) \pi^{-}
$$

Then the dressing transform $\hat{S}$ of $S$ by $R$ is given by

$$
\hat{S}=T_{0} S T_{0}^{-1}
$$

Moreover, if $S$ is the central sphere congruence of a constrained Willmore surface $L$ with multiplier $q$, then the dressing transform $\hat{L}$ and its multiplier $\hat{q}$ are given by:

$$
\begin{aligned}
& \hat{L}=T_{0} L, \\
& \hat{q}=T_{0} q T_{0}^{-1} .
\end{aligned}
$$

Proof. The only thing to check here is that $T_{0}$ so defined is indeed an isomorphism. But the image of $T_{0}$ is $\hat{S}^{+}+\hat{S}^{-}=\underline{\mathbb{C}}^{4}$ thanks to (4.21).

Clearly there is some gauge freedom here: we could precompose $T_{0}$ with any gauge transformation, which is a scalar multiple of the identity on each of $S^{ \pm}$. We shall exploit this below.

\subsection{Darboux transforms and Riccati equations}

A transformation of Willmore surfaces in $S^{4}$ via solutions of a Riccati equation is described in [10, Chapter 12] while a related transform is derived by Leschke in [26]. We now show that these transforms all amount to untwisted dressing by simple factors as described in Section 4.4. Along the way, we extend the theory to constrained Willmore surfaces.

For this, we specialize the considerations of the last section to the case where the dressing gauge $R$ is a simple factor $P_{\nu, M}$. Recall that here $\nu \in \mathbb{C}^{\times} \backslash$ $S^{1}$ and $M$ is a $\nabla^{\nu}$-parallel null line subbundle with $M, \bar{M}$ complementary. Equivalently, in the quaternionic formalism, $M=\wedge^{2} W$ for $W$ a $\nabla^{\nu}$-parallel complex subbundle of $\underline{\mathbb{C}}^{4}$ such that $\underline{\mathbb{C}}^{4}=W \oplus j W$ (so that rank $W=2$ ). If 
we now define $\Gamma_{j W}^{W}(\mu) \in \Gamma \operatorname{End}\left(\underline{\mathbb{C}}^{4}\right)$ by

$$
\Gamma_{j W}^{W}(\mu)= \begin{cases}\mu & \text { on } W \\ 1 / \mu & \text { on } j W\end{cases}
$$

then

$$
\widehat{P_{\nu, M}(\mu)}=\Gamma_{j W}^{W}\left(\sqrt{\frac{\left(1-\nu^{*}\right)(\mu-\nu)}{(1-\nu)\left(\mu-\nu^{*}\right)}}\right) .
$$

We now have the following improvement on Corollary 4.13 in case that $R$ is a simple factor:

Theorem 4.14. Let $\left(S, \nabla^{\mu}\right)$ be k-perturbed harmonic in $\underline{\mathbb{C}}^{6}$. Fix $\nu \in \mathbb{C}^{\times}$ $S^{1}$ and let $W \leq \mathbb{C}^{4}$ be a $\nabla^{\nu}$-parallel subbundle such that $W \oplus j W=\underline{\mathbb{C}}^{4}$. Define $\mu_{0}, \mu_{1} \in \mathbb{C}^{\times}$by

$$
\mu_{0}=i \frac{\nu+1}{\nu-1}, \quad \mu_{1}=i \frac{\nu^{*}+1}{\nu^{*}-1} .
$$

Further, define $X \in \Gamma \mathfrak{g l}\left(\underline{\mathbb{H}}^{2}\right)$ by

$$
X= \begin{cases}\mu_{0} & \text { on } W \\ \mu_{1} & \text { on } j W\end{cases}
$$

and then $T \in \Gamma \mathfrak{g l}\left(\underline{\mathbb{H}}^{2}\right)$ by

$$
T=X-S
$$

Then $P_{\nu, \wedge^{2} W}$ is an untwisted dressing gauge for $\left(S, \nabla^{\mu}\right)$ on the open set on which $T$ is an isomorphism and the dressing transform of $S$ by $P_{\nu, \wedge^{2} W}$ is given by

$$
\hat{S}=T S T^{-1}
$$

In particular, $\hat{S}$ is $k$-perturbed harmonic.

Moreover, when $S$ is the central sphere congruence of a constrained Willmore surface $L$ with multiplier $q$, then the untwisted dressing transform of $L$ by $P_{\nu, \wedge^{2} W}$ is given by

$$
\begin{aligned}
\hat{L} & =T L, \\
\hat{q} & =T q T^{-1} .
\end{aligned}
$$


Proof. Set $R=P_{\nu, \wedge^{2} W}$. We claim that there are constants $\lambda_{0}, \lambda_{1} \in \mathbb{C}^{\times}$such that:

$$
\widetilde{R}(0)=\lambda_{0}(X+i) \quad \widetilde{R}(\infty)=\lambda_{1}(X-i)
$$

Given the claim, we immediately deduce that:

$$
\begin{aligned}
\widetilde{R}(0)_{\mid S^{-}} & =\lambda_{0}(X+i)_{\mid S^{-}}=\lambda_{0} T_{\mid S^{-}}, \\
\widetilde{R}(\infty)_{\mid S^{+}} & =\lambda_{1}(X-i)_{\mid S^{+}}=\lambda_{1} T_{\mid S^{+}}
\end{aligned}
$$

We note that $\mu_{1}=-\overline{\mu_{0}}$ so that $X$ and so $T$ are $\mathfrak{g l}\left(\underline{\mathbb{H}}^{2}\right)$-valued. Moreover, $T$ is an isomorphism exactly when $\widetilde{R}(0) S^{-} \cap \widetilde{R}(\infty) S^{+}=\{0\}$ or, equivalently, when $R(0) V_{-}^{\perp}$ and $R(\infty) V_{+}^{\perp}$ are complementary, that is, when $R$ is an untwisted dressing gauge. The rest of the theorem now follows at once from Proposition 4.12.

For the claim, note that both sides of each equation in (4.25) have $W$ and $j W$ as eigenspaces and so we must simply equate eigenvalues and require that

$$
\begin{aligned}
& \sqrt{\frac{\nu\left(1-\nu^{*}\right)}{\nu^{*}(1-\nu)}}=\lambda_{0}\left(\mu_{0}+i\right) \quad \sqrt{\frac{1-\nu^{*}}{1-\nu}}=\lambda_{1}\left(\mu_{0}-i\right), \\
& \sqrt{\frac{\nu^{*}(1-\nu)}{\nu\left(1-\nu^{*}\right)}}=\lambda_{0}\left(\mu_{1}+i\right) \quad \sqrt{\frac{1-\nu}{1-\nu^{*}}}=\lambda_{1}\left(\mu_{i}-i\right) .
\end{aligned}
$$

These amount to linear equations for the $\mu_{i}$ which are solved by (4.22) and then the $\lambda_{i}$ are given by

$$
\lambda_{0}=-\frac{i}{2} \sqrt{\frac{(\nu-1)\left(\nu^{*}-1\right)}{\nu \nu^{*}}} \quad \lambda_{1}=-\frac{i}{2} \sqrt{(\nu-1)\left(\nu^{*}-1\right)} .
$$

Remark 4.15. For Willmore $L$ and, more generally, harmonic $S$, the construction in Theorem 4.14 of $\hat{S}$ and $\hat{L}$ from a $\nabla^{\nu}$-parallel $W$ coincides with Leschke's $\nu$-Darboux transform [26, Theorems 4.2 and 4.4]. She conjectured that this procedure should amount to a dressing transform as we have just affirmed.

Theorem 4.14 offers a different perspective on untwisted dressing by simple factors by focusing on the field of endomorphisms $X$ rather than 
the dressing gauge $P_{\nu, \wedge^{2} W}$. The key conditions on $X$, that it have constant eigenvalues and $\nabla^{\nu}$ or $\nabla^{\nu^{*}}$-parallel eigenspaces $W, j W$, are encapsulated in a Riccati equation with a conserved quantity:

Proposition 4.16. Let $\left(S, \nabla^{\mu}\right)$ be $k$-perturbed harmonic, $X \in \Gamma \mathfrak{g l}\left(\underline{\mathbb{H}}^{2}\right)$ and $\mu_{0} \in \mathbb{C} \backslash \mathbb{R}$. Then $X$ has eigenvalue $\mu_{0}$ with rank $2 \nabla^{\nu}$-parallel eigenbundle if and only if

$\left(\mu_{1}-\mu_{0}\right) d X=X\left(\beta^{*}-\beta\right) X+\left(\mu_{0} \beta-\mu_{1} \beta^{*}\right) X+X\left(\mu_{1} \beta-\mu_{0} \beta^{*}\right)+\mu_{0} \mu_{1}\left(\beta^{*}-\beta\right)$

$$
X^{2}-\left(\mu_{0}+\mu_{1}\right) X+\mu_{0} \mu_{1}=0 \text {, }
$$

where $\mu_{1}=\overline{\mu_{0}}, \nabla^{\nu}=d+\beta$ and $\nabla^{\nu^{*}}=d+\beta^{*}$.

Proof. First note that if $X$ has rank $2 \mu_{0}$-eigenbundle $W$ then it also has $\mu_{1}$-eigenbundle $j W$ with $\underline{\mathbb{C}}^{4}=W \oplus j W$ so that the minimal polynomial of $X$ is $X^{2}-\left(\mu_{0}+\mu_{1}\right) X+\mu_{0} \mu_{1}$. Conversely, if $X$ solves $(4.26 \mathrm{~b})$ with $\mu_{0}$ nonreal, then, since $[X, j]=0, X$ is not a scalar multiple of the identity and so has $X^{2}-\left(\mu_{0}+\mu_{1}\right) X+\mu_{0} \mu_{1}$ as its minimal polynomial. It follows at once that $X$ has eigenvalues $\mu_{0}, \mu_{1}$ with rank 2 eigenbundles.

Thus, we may assume that $X$ has rank $2 \mu_{0}$-eigenbundle $W$ and the only remaining issue is whether $W$ is $\nabla^{\nu}$-parallel, or equivalently, $j W$ is $\nabla \nu^{*}$-parallel. This is easily seen to be equivalent to the demand that

$$
\begin{aligned}
\left(X-\mu_{0}\right) \nabla^{\nu}\left(X-\mu_{1}\right) & =0, \\
\left(X-\mu_{1}\right) \nabla^{\nu^{*}}\left(X-\mu_{0}\right) & =0,
\end{aligned}
$$

or, since these last have image in different eigenbundles,

$$
\left(X-\mu_{0}\right) \nabla^{\nu}\left(X-\mu_{1}\right)=\left(X-\mu_{1}\right) \nabla^{\nu^{*}}\left(X-\mu_{0}\right) \text {. }
$$

However, writing this out in terms of $\beta$ and $\beta^{*}$ promptly yields (4.26a).

Remark 4.17. In the situation of Theorem 4.14 with $\mu_{0}$ given by (4.22), we see that the excluded case that $\mu_{0} \in \mathbb{R}$ amounts to the already excluded case of $\nu \in S^{1}$.

Observe that the coefficients in the Riccati equation (4.26a) are all pure imaginary so that solutions with initial condition in $\mathfrak{g l}\left(\underline{\mathbb{H}}^{2}\right)$ remain in $\mathfrak{g l}\left(\underline{\mathbb{H}}^{2}\right)$. In fact, much more is true: the Riccati equation is completely integrable and admits (4.26b) as a first integral. All this uses almost nothing about the 
specifics of the situation beyond the flatness of the connections $\nabla^{\nu}$ and $\nabla^{\nu^{*}}$. Indeed, we have:

Proposition 4.18. Let $\mathrm{A}$ be a complex finite-dimensional associative algebra with unit, $\mu_{0} \neq \mu_{1} \in \mathbb{C}$ and $\beta, \beta^{*}$ A-valued 1 -forms on $\Sigma$ defining flat connections:

$$
d \beta+\beta \wedge \beta=d \beta^{*}+\beta^{*} \wedge \beta^{*}=0 .
$$

Then the Riccati equation (4.26a)

$$
\begin{aligned}
\left(\mu_{1}-\mu_{0}\right) d X= & X\left(\beta^{*}-\beta\right) X+\left(\mu_{0} \beta-\mu_{1} \beta^{*}\right) X \\
& +X\left(\mu_{1} \beta-\mu_{0} \beta^{*}\right)+\mu_{0} \mu_{1}\left(\beta^{*}-\beta\right)
\end{aligned}
$$

for $X: \Sigma \rightarrow \mathrm{A}$ is completely integrable.

Moreover, if $X$ is a solution and $I=X^{2}-\left(\mu_{0}+\mu_{1}\right) X+\mu_{0} \mu_{1}$, then $I$ solves the linear equation

$\left(\mu_{1}-\mu_{0}\right) d I=I\left(\beta^{*}-\beta\right) X+X\left(\beta^{*}-\beta\right) I+I\left(\mu_{1} \beta-\mu_{0} \beta^{*}\right)+\left(\mu_{0} \beta-\mu_{1} \beta^{*}\right) I$

and so, in particular, vanishes identically if it vanishes at a single point.

Proof. One can prove complete integrability by direct computation but we offer a more conceptual and, perhaps, instructive proof based on the well known classical observation that Riccati equations are the affine expression of linear equations in homogeneous coordinates. So consider a general Riccati equation

$$
d X=X A X+B X+X C+D
$$

with A-valued 1-forms $A, B, C, D$. Write $X=P Q^{-1}$ for $P, Q: \Sigma \rightarrow$ A (we can always do this near some initial point $p_{0} \in \Sigma$ by taking $\left.Q\left(p_{0}\right)=1\right)$. Then the equation becomes

$$
d P-B P-D Q-P Q^{-1}(d Q+A P+C Q)=0,
$$

so that integrability is guaranteed by flatness of the connection on the trivial $\mathrm{A} \oplus \mathrm{A}$ bundle given by

$$
d+\left(\begin{array}{cc}
-B & -D \\
A & C
\end{array}\right)
$$

In the case at hand, this connection reads

$$
d+\frac{1}{\mu_{1}-\mu_{0}}\left(\begin{array}{cc}
\mu_{1} \beta^{*}-\mu_{0} \beta & \mu_{0} \mu_{1}\left(\beta-\beta^{*}\right) \\
\beta^{*}-\beta & \mu_{1} \beta-\mu_{0} \beta^{*}
\end{array}\right)=\left(\begin{array}{cc}
\mu_{0} & \mu_{1} \\
1 & 1
\end{array}\right) \cdot\left(d+\left(\begin{array}{cc}
\beta & 0 \\
0 & \beta^{*}
\end{array}\right)\right)
$$


which is clearly flat.

The derivation of the linear equation for $I$ is a straightforward computation which we leave to the interested reader.

Let us summarize this development which amounts to a construction of new $k$-perturbed harmonic bundles from old via solving a Riccati equation:

Corollary 4.19. Let $\left(S, \nabla^{\mu}\right)$ be k-perturbed harmonic in $\underline{\mathbb{C}}^{6}$ and fix $\nu \in$ $\mathbb{C}^{\times} \backslash S^{1}$. Define $\mu_{0}, \mu_{1} \in \mathbb{C}^{\times}$by (4.22). Fix a base-point $x_{o} \in \Sigma$ and $X_{o} \in$ $\mathfrak{g l}\left(\mathbb{H}^{2}\right)$ satisfying $X_{o}^{2}-\left(\mu_{0}+\mu_{1}\right) X_{o}+\mu_{0} \mu_{1}=0$.

There is (locally) $X \in \Gamma \mathfrak{g l}\left(\underline{\mathbb{H}}^{2}\right)$ solving:

$$
\begin{aligned}
\left(\mu_{1}-\mu_{0}\right) d X= & X\left(\beta^{*}-\beta\right) X+\left(\mu_{0} \beta-\mu_{1} \beta^{*}\right) X \\
& +X\left(\mu_{1} \beta-\mu_{0} \beta^{*}\right)+\mu_{0} \mu_{1}\left(\beta^{*}-\beta\right) \\
X\left(x_{o}\right)= & X_{o}
\end{aligned}
$$

where $\nabla^{\nu}=d+\beta$ and $\nabla^{\mu}=d+\beta^{*}$. Then, with $T=X-S, \hat{S}=T S T^{-1}$ is $k$-perturbed harmonic on the open set on which $T$ is invertible.

Moreover, if $S$ is the central sphere congruence of a constrained Willmore surface $L$, then $\hat{S}$ is the central sphere congruence of a constrained Willmore surface $T L$.

Of course, our Riccati equation for $X$ can be phrased as a Riccati equation for $T$ the form of which, for general $\nu$, is not very edifying. However, when $\nu$ is real, matters simplify considerably and we not only recover the Darboux transforms of Willmore surfaces described in [10, Section 12.3] but generalize them to the constrained Willmore case.

So let $L$ be a constrained Willmore surface with multiplier $q$ and central sphere congruence $S$. We then have $\nabla^{\mu}=d+(\mu-1)\left(\mathcal{A}_{S}+q\right)^{1,0}+\left(\mu^{-1}-\right.$ 1) $\left(\mathcal{A}_{S}+q\right)^{0,1}$. Fix $\nu \in \mathbb{R} \backslash\{ \pm 1\}$ so that $\nu^{*}=1 / \nu$ and then

$$
\mu_{0}=i \frac{\nu+1}{\nu-1}=-\mu_{1}
$$

Set

$$
\rho=-\frac{(\nu-1)^{2}}{4 \nu}=\frac{2-\nu-\nu^{*}}{4}
$$

so that

$$
\rho^{-1}-1=\mu_{0}^{2}=\mu_{1}^{2}
$$


We may now use (4.18a) along with (4.17) and (4.20b) to write the Riccati equation (4.26a) for $X$ as the following Riccati equation for $T=X-S$ :

$$
d T=\rho T\left(2 *\left(\mathcal{A}_{S}+q\right)\right) T-4 \rho T q-2 *\left(\mathcal{Q}_{S}+q\right)
$$

with first integral $(T+S)^{2}-\left(\rho^{-1}-1\right)$.

In this situation, $\rho^{-1}<1$ and, conversely, if $\rho^{-1}<1$, we can rearrange (4.27) and recover $\nu, \nu^{*}$ as the two real roots of $\mu^{2}+2(2 \rho-1) \mu+1=0$. We therefore have:

Theorem 4.20. Let $L$ be a constrained Willmore surface in $S^{4}$ with multiplier $q$ and central sphere congruence $S$. Let $\rho \in \mathbb{R}$ with $\rho^{-1}<1$ and let $T \in \mathfrak{g l}\left(\underline{\mathbb{H}}^{2}\right)$ be a solution of the integrable Riccati equation (4.28) with $(T+$ $S)^{2}-\left(\rho^{-1}-1\right)=0$ at one, and hence every, point.

Then $\hat{L}=T L$ is also a constrained Willmore surface with multiplier $T q T^{-1}$ and central sphere congruence $T S T^{-1}$ on the open set on which $T$ is invertible.

Moreover, $\hat{L}$ is an untwisted dressing transform of $L$ by a simple factor.

For Willmore surfaces (thus $q=0$ ), this construction of $\hat{L}$ is the Darboux transform of [10, Section 12.3] with the caveat that their Riccati equation swops the roles of $\mathcal{A}_{S}$ and $\mathcal{Q}_{S}$ and their $T$ is the inverse of ours. This is, of course, a well-known symmetry of the Riccati equation.

\section{Acknowledgments}

The first-named author gratefully acknowledges instructive conversations with David Calderbank, Katrin Leschke and Udo Hertrich-Jeromin. The second-named author was supported by Fundação para a Ciência e a Tecnologia, Portugal, with the PhD scholarship SFRH/BD/6356/2001, and by Fundação da Universidade de Lisboa, with the postdoctoral scholarship CMAF-BPD-01/09.

\section{References}

[1] W. Blaschke, Vorlesungen über differentialgeometrie III, Grundlehren Math., 29, Springer, Berlin, 1929.

[2] C. Bohle, Constrained Willmore tori in the 4-sphere, J. Differ. Geom., 86(1) (2010), 71-131. 
[3] C. Bohle, F. Pedit and U. Pinkall, The spectral curve of a quaternionic holomorphic line bundle over a 2-torus, Manuscr. Math., 130(3) (2009), 311-352.

[4] C. Bohle, G.P. Peters and U. Pinkall, Constrained Willmore surfaces, Calc. Var. Partial Differ. Equ., 32(2) (2008), 263-277.

[5] D. Brander and J. Dorfmeister, Generalized DPW method and an application to isometric immersions of space forms, Math. Z., 262(1) (2009), 143-172.

[6] R.L. Bryant, Conformal and minimal immersions of compact surfaces into the 4-sphere, J. Differ. Geom., 17(3) (1982), 455-473.

[7] R.L. Bryant, A duality theorem for Willmore surfaces, J. Differ. Geom., 20(1) (1984), 23-53.

[8] F.E. Burstall, Isothermic surfaces: conformal geometry, Clifford algebras and integrable systems, (2006), Integrable systems, geometry, and topology, AMS/IP Stud. Adv. Math., 36, Amer. Math. Soc., Providence, RI, 1-82.

[9] F.E. Burstall, J.F. Dorfmeister, K. Leschke and A.C. Quintino, Darboux transforms and simple factor dressing of constant mean curvature surfaces, Manuscr. Math., 140(1-2) (2013), 213-236.

[10] F.E. Burstall, D. Ferus, K. Leschke, F. Pedit and U. Pinkall, Conformal geometry of surfaces in $S^{4}$ and quaternions, Lecture Notes in Mathematics, Springer-Verlag, Berlin, 2002, 1772, ISBN:3-540-43008-3

[11] F.E. Burstall, D. Ferus, F. Pedit and U. Pinkall, Harmonic tori in symmetric spaces and commuting Hamiltonian systems on loop algebras, Ann. Math. (2), 138(1) (1993), 173-212.

[12] F.E. Burstall and D. Calderbank, Conformal submanifold geometry IV$V$, In preparation.

[13] F.E. Burstall and D.M.J. Calderbank, Conformal submanifold geometry I-III, 2010, arXiv:1006.5700 [math.DG].

[14] Burstall, Francis, F. Pedit and U. Pinkall, Schwarzian derivatives and flows of surfaces, (2002), Differential Geometry and Integrable Systems Tokyo, 2000, Contemporary Mathematics Series, 308, Amer. Math. Soc., Providence, RI, 39-61. 
[15] F.E. Burstall, N.M. Donaldson, F. Pedit and U. Pinkall, Isothermic submanifolds of symmetric R-spaces, J. Reine Angew. Math., 660 (2011), 191-243.

[16] G. Darboux, Leçons sur la théorie générale des surfaces et les applications géométriques du calcul infinitésimal. Parts 1 and 2, GauthierVillars, Paris, 1887.

[17] J. Eells and S. Salamon, Twistorial construction of harmonic maps of surfaces into four-manifolds, (1985), Ann. Scuola Norm. Sup. Pisa Cl. Sci. (4), 12(4) (1986), 589-640.

[18] N. Ejiri, Willmore surfaces with a duality in $S^{N}(1)$, Proc. Lond. Math. Soc. (3), $\mathbf{5 7}(2)$ (1988), 383-416.

[19] D. Ferus, K. Leschke, F. Pedit and U. Pinkall, Quaternionic holomorphic geometry: Plücker formula, Dirac eigenvalue estimates and energy estimates of harmonic 2-tori, Invent. Math., 146(3) (2001), 507-593.

[20] T. Friedrich, The geometry of t-holomorphic surfaces in $S^{4}$, Math. Nachr., 137 (1988), 49-62.

[21] S. Germain, Mémoire sur la coubure des surfaces, Crelle’s J., 7 (1831), 1-29.

[22] U. Hertrich-Jeromin, Introduction to Möbius differential geometry, London Mathematical Society Lecture Note Series, 2003, 300, Cambridge University Press, Cambridge, ISBN:0-521-53569-7.

[23] N.J. Hitchin, Harmonic maps from a 2-torus to the 3-sphere, J. Differ. Geom., 31(3) (1990), 627-710.

[24] I. Khemar, Elliptic integrable systems: a comprehensive geometric interpretation, Mem. Amer. Math. Soc., 219(1031) (2012), x+217.

[25] S. Kobayashi, and J.-I. Inoguchi, Characterizations of BianchiBäcklund transformations of constant mean curvature surfaces, Int. J. Math., 16(2) (2005), 101-110.

[26] K. Leschke, Harmonic map methods for Willmore surfaces, Harmonic maps and differential geometry, Contemporary Mathematics Series, 542, Amer. Math. Soc., Providence, RI, 2011, 203-212.

[27] A. Mahler, Bianchi-Bäcklund transforms for constant mean curvature surfaces with umbilics. Theory and applications, 2002, Toledo PhD thesis. 
[28] F.C. Marques, A. Neves, Min-Max theory and the Willmore conjecture, (2012), 95, arXiv:1202.6036 [math.DG]

[29] R. Pacheco, Bianchi-Bäcklund transforms and dressing actions, revisited, Geom. Dedicata, 146 (2010), 85-99.

[30] K. Pohlmeyer, Integrable Hamiltonian systems and interactions through quadratic constraints, Commun. Math. Phys., 46(3) (1976), $207-221$.

[31] A.C. Quintino, Constrained Willmore surfaces: symmetries of a Möbius invariant integrable system, PhD thesis, University of Bath, 2008.

[32] A.C. Quintino, Constrained Willmore surfaces: symmetries of a Möbius invariant integrable system-based on the author's PhD thesis, 2009, arXiv:0912.5402v1 [math.DG].

[33] A.C. Quintino, Spectral deformation and Bäcklund transformation of constrained Willmore surfaces, Differ. Geom. Appl., 29(Suppl. 1) (2011), S261-S270.

[34] J. Richter, Conformal maps of a Riemannian surface onto the space of quaternions, PhD thesis, Berlin, 1997.

[35] M. Rigoli, The conformal Gauss map of submanifolds of the Möbius space, Ann. Global Anal. Geom., 5(2) (1987), 97-116.

[36] B.A. Rozenfel'd, Conformal differential geometry of families of $C_{m}$ in $C_{n}$, Mat. Sbornik N.S., 23(65) (1948), 297-313.

[37] C.-L. Terng, Geometries and symmetries of soliton equations and integrable elliptic equations Surveys on geometry and integrable systems, Adv. Stud. Pure Math., 51, Math. Soc. Japan, Tokyo, 2008, 401-488.

[38] C.-L. Terng and K. Uhlenbeck, Bäcklund transformations and loop group actions, Commun. Pure Appl. Math., 53(1) (2000), 1-75.

[39] G. Thomsen, Ueber konforme Geometrie I: Grundlagen der konformen Flaechentheorie, Hamb. Math. Abh., 3 (1923), 31-56.

[40] K. Uhlenbeck, Harmonic maps into Lie groups: classical solutions of the chiral model, J. Differ. Geom., 30(1) (1989), 1-50.

[41] T.J. Willmore, Note on embedded surfaces, An. Şti. Univ. "Al. I. Cuza" Iaşi Sect. I a Mat. (N.S.), 11B (1965), 493-496. 
[42] V.E. Zaharov and A.B. Šabat, Integration of the nonlinear equations of mathematical physics by the method of the inverse scattering problem. II, Funktsional. Anal. i Prilozhen., 13(3) (1979), 13-22.

[43] V.E. Zakharov and A.V. Mikhaulov, Relativistically invariant twodimensional models of field theory which are integrable by means of the inverse scattering problem method, Russian language, Sov. Phys. J. Exp. Theor. Phys. , 74(6) (1978), 1017-1027 (1979).

Department of Mathematical Sciences

UNIVERSITY OF BATH

BATH BA2 7AY

$\mathrm{UK}$

E-mail address: feb@maths.bath.ac.uk

Centro de Matemática e AplicaÇões Fundamentais da Universidade de LISBOA

Avenida Professor Gama Pinto, 2

1649-003 LisBoA

PORTUGAL

E-mail address: aurea@ptmat.fc.ul.pt

Received July 10, 2013 